\title{
AN ANALYSIS OF 700 CASES OF CARCINOMA OF THE HYPOPHARYNX, THE OESOPHAGUS, AND THE PROXIMAL STOMACH
}

\author{
BY \\ B. T. LE ROUX \\ From the Department of Clinical Surgery, University of Edinburgh
}

(RECEIVED FOR PUBLICATION JANUARY 1, 1961)

In the course of 12 years, 1,200 patients have been investigated for dysphagia in the Thoracic Surgical Unit in Edinburgh. Seven hundred of these patients were shown to have as the cause of their dysphagia a carcinoma of the hypopharynx, of the oesophagus, or of the proximal part of the stomach. All these patients were referred for investigation because of dysphagia, and dysphagia is the link between them. The review has been undertaken at this time because 700 is a convenient number and one sufficiently large to lend some weight to the analysis.

The Unit in Edinburgh serves a relatively closed population of about one and a half million. Most of the patients with dysphagia in the south-eastern region of Scotland are referred to this Unit for investigation. A sprinkling of patients with dysphagia comes from further north and further south, but these probably compensate for the small number with oesophageal carcinoma occurring within this region and not referred to this Unit. The number of patients investigated, therefore, probably represents fairly accurately the incidence of proximal alimentary carcinoma in this population. A further advantage of the analysis is that the management of patients with carcinomatous dysphagia has been uniform throughout the period under review, so that the review is an accurate criticism of a standard management and a standard operation in the hands of a small number of surgeons, all of them using the same technique.

In 20 patients, $3 \%$ of the total, the cause of the dysphagia was a squamous carcinoma of the hypopharynx (laryngopharynx); 19 of these patients were women. In 382 patients, $54.5 \%$ of the total, the cause of the dysphagia was a squamous carcinoma of the oesophagus; approximately three-fifths of these patients were men. In 26 patients, just under $4 \%$ of the total, the carcinoma was undifferentiated and in the oesophagus; in this group the sexes were equally represented. In 음 the remaining 272 patients, just under $39 \%$ of $\vec{C}$ the total, the cause of the dysphagia was an adenocarcinoma, either visible oesophagoscopically, or in the proximal part of the stomach beyond the range of the oesophagoscope. An analysis of these 700 cases outlines the natural history of carcinoma of the proximal alimentary tract, and shows what influence resection in the hands of this Unit may have in altering the natural course of the disease.

In this analysis adenocarcinoma and squamous carcinoma are discussed separately. Adeno? carcinoma is probably primarily a gastric tumour, and all the squamous carcinomas in this series were in the oesophagus or hypopharynx. The undifferentiated carcinomas were all in the oesophagus and did not behave in a way importantly different from the squamous tumours. Many of the squamous carcinomas involved the cardia, and many of the adenocarcinomas extended into the oesophagus, some as high as the aortic arch. But, while many squamous carcinomas in the middle third of the oesophagus had distal to them normal squamous epithelium, no adenocarcinoma was found in the middle third of the oesophagus with squamous epithelium distal to it, unless this was a submucous lymphatic metastasis from a more distal tumour. There is also a significant difference in the direction of spread of squamous and adenocarcinoma, a consequence of site of occurrence, the cell type probably being unimportant.

In assessing operability and operative results, the oesophageal tumours are called high or low. A high tumour is one $(a)$ which was approached from the right, or $(b)$ if approached from the left necessitated the making of an anastomosis above the level of the aortic arch, or $(c)$ if a resection was not undertaken, one the proximal edge of 
which lay closer than $30 \mathrm{~cm}$. to the upper alveolus when seen through the oesophagoscope. When a low tumour was resected the anastomosis lay below the level of the aortic arch, and the proximal limit of an irresectable low tumour was $30 \mathrm{~cm}$. or further from the upper alveolus.

The frequency of the different tumours is shown in Table I. In 271 patients a resection was not

TABLE I

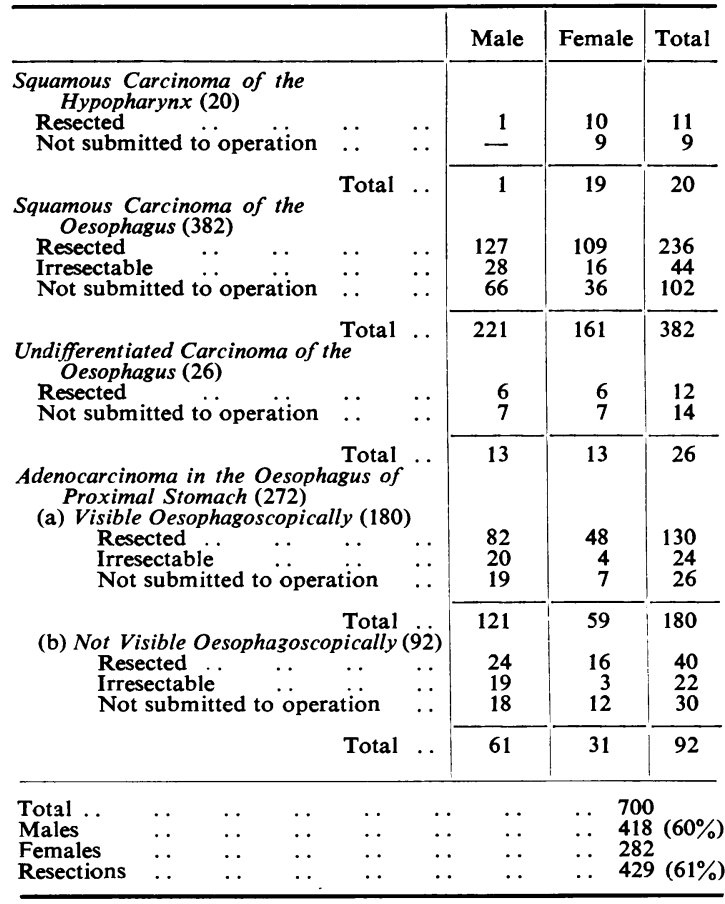

attempted or, if attempted, was not completed, an incidence of inoperability of $39 \%$. Four hundred and twenty-nine resections were undertaken $(61 \%)$. None of the 11 patients from whom a carcinoma of the hypopharynx was resected died from operation. The operative mortality in the remaining 418 patients, all of whom had an oesophago-gastrectomy, was $30 \%$. This mortality is analysed in a subsequent paper (le Roux, 1962a), and a further paper (le Roux, 1962b) is devoted to haemorrhage complicating the late convalescence of patients who had undergone oesophagogastrectomy.

The investigation of these 700 patients included radiography with barium, and endoscopy. The endoscopic instruments used were the laryngoscope only in some with tumours in the hypopharynx, and the Negus oesophagoscope in all others. A gastroscope was not used. When an adenocarcinoma of the proximal stomach is described as beyond the range of endoscopic examination, this means that it was not visible through the long $(45 \mathrm{~cm}$.) adult Negus oesophagoscope.

\section{Souamous Carcinoma of the HypopharynX}

Twenty patients with dysphagia were shown to have a squamous carcinoma of the hypopharynx. The age range is shown in Fig. 1. Nineteen of the 20 patients were women. This preponderance of women is usual in carcinoma of the hypopharynx, and this is the only primary malignant tumour of the proximal alimentary tract with a higher incidence amongst women than amongst men.

Not RESECTED.-Nine women were found to have cervical glandular metastases when they were first seen. In three of these patients the tumour was irradiated (Fig. 3). None survived longer than seven months from the time of admission, and irradiation had no appreciable influence in the prolongation of life or in the restoration of swallowing. Four of these nine patients had a long history of intermittent dysphagia, one of them for 30 years and the others for more than 20 years. They were anaemic and these are probably examples of sideropenic dysphagia. The character of the dysphagia had changed in these four patients two to six months before they were referred for advice. The average survival from the onset of dysphagia, or from the change in character of dysphagia, in these nine patients was seven months, and the average duration of

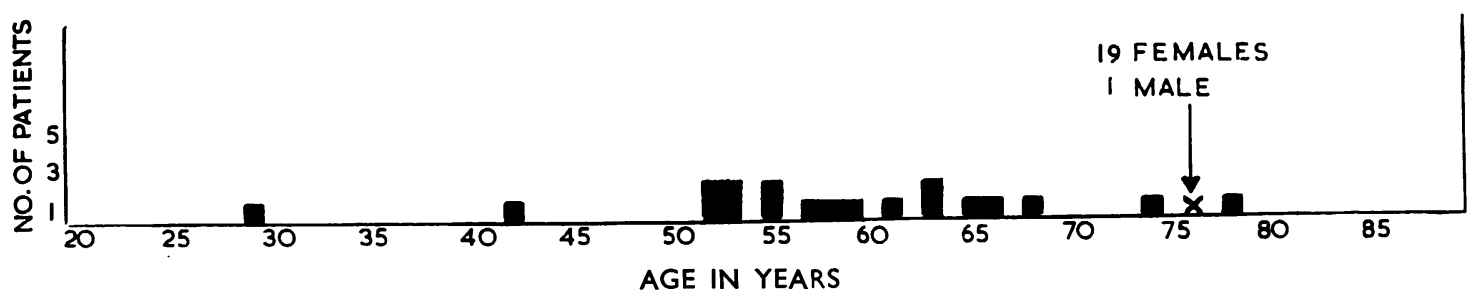

FIG. 1.-Age range in patients with squamous carcinoma of the hypopharynx. 


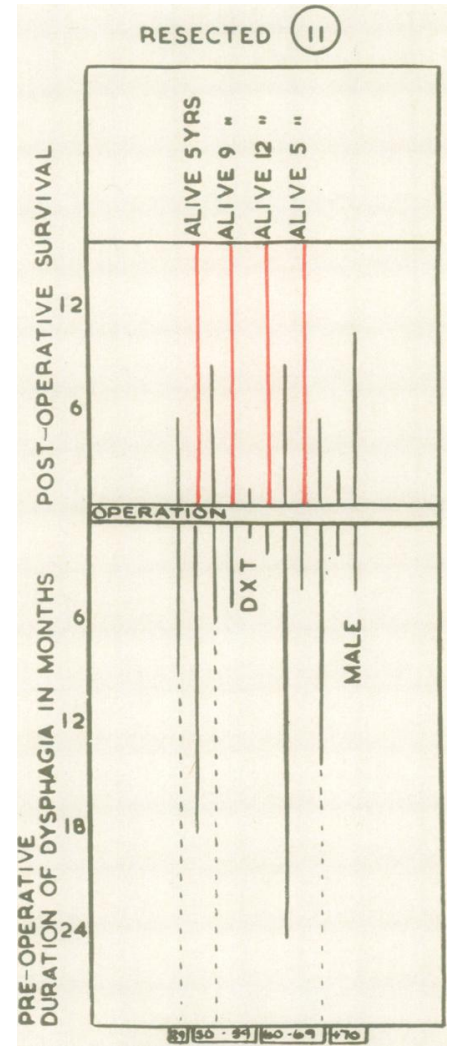

FIG. 2 .

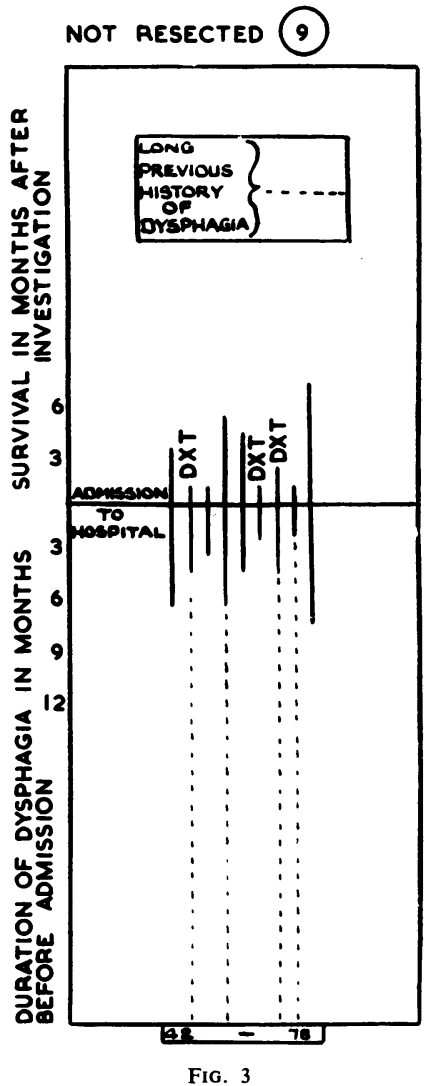

FIGS. 2 and 3.-Each vertical line represents a patient, and these are arranged the youngest on the left and the oldest on the right. (DXT is an abbreviation for deep $x$-ray therapy.)

dysphagia before admission to hospital was four months. Death in eight was due to aspiration pneumonia and in one to haemorrhage.

RESECTED.-Ten women and one man were submitted to resection (Fig. 2): lateral pharyngectomy or cervical oesophagectomy in four and laryngopharyngectomy in seven, leaving these with a permanent tracheotomy. Four patients, all women, survived long enough for the satisfactory fashioning of a cervical oesophagus from skin, and these four patients are alive, 12 years, nine years, and five years (two patients) after resection. They all swallow normally, one requiring dilatation of the skin tube at about yearly intervals. The man and two women died from recurrence of tumour within a year of resection, one patient died from aspiration pneumonia two months after resection, and three patients died from haemorrhage five weeks to five months after resection. In two, haemorrhage was from erosion of the right common carotid artery by tumour recurrence; in the third, haemorrhage was from the nasopharynx, where residual tumour was found. Three of these patients, all women and all anaemic, had a long previous history of intermittent dysphagia (15 to 20 years), a change in the character of the dysphagia precipitating admission. None of these survived operation by more than eight months. In three of the four patients alive after resection, dysphagia had been present for less than five months before resection, but in the fourth survivor dysphagia had been present for 18 months. One patient dying from tumour recurrence had had dysphagia for two years before resection.

The average duration of dysphagia in these 11 ? patients was eight months, from onset in those without a very long history, or from changing character in those three with a very long history. Average survival from the onset of dysphagia in those dying from recurrence, pneumonia, or haemorrhage was 14 months. 
In the case of one woman a carcinoma of the hypopharynx was irradiated 14 months before she returned with a recurrence of dysphagia for which she was submitted to resection. She had had dysphagia for a month before she was irradiated; irradiation improved her swallowing but the tumour remained visible; she had nearly total dysphagia for a month before requesting further treatment; she died five weeks after operation from haemorrhage from the nasopharynx.

To sum up, of 20 patients with carcinoma of the hypopharynx, 19 of them women, nine had metastases when first seen and these patients died on an average seven months from the onset of dysphagia. Eleven were submitted to operation and four of these $(36 \%)$ are alive more than five years later. Seven of the 20 patients had sideropenic dysphagia for many years, and all seven succumbed rapidly.

\section{Squamous Carcinoma of the Oesophagus}

Three hundred and eighty-two patients with dysphagia were shown to have a squamous carcinoma of the oesophagus. The age and sex distribution of these patients are shown in Fig. 4. The ratio of men to women is about $3: 2$. The oesophageal carcinoma was resected in 236 patients $(62 \%)$. Forty-four patients $(12 \%)$ were submitted to thoraco-laparotomy with a view to resection but were found to have either an irresectable tumour or one from which dissemination was so gross that resection was unjustified because of relatively mild dysphagia. In 102 patients $(26 \%)$ thoracotomy was not undertaken because they had metastases when first seen. or because they were too old or too ill or declined operation. Thoraco-laparotomy was undertaken in five patients in the knowledge that the tumour had disseminated and resection was completed in four of these. In those patients in whom resection was not undertaken attempts to improve swallowing included the use of stomach or jejunum to bypass the tumour. intubation of the oesophageal stricture with a Simmonds' or Souttar's tube, and irradiation using either deep radiotherapy or radium bougies. The percentage of patients with squamous carcinoma of the oesophagus found to have a resectable tumour was the same as amongst patients with adenocarcinoma.

Squamols Carcinoma of the Oesophagus not Treated by Operation.-One hundred and two patients with squamous carcinoma of the oesophagus were not submitted to operation. Sixty-six of these were men and 36 were women. The youngest patient was 47 and the oldest 86 . The age incidence in the two sexes is shown in Fig. 5. All these patients are dead. In all of them the tumour was visible endoscopically, and histological confirmation of the diagnosis was obtained by biopsy of tissue obtained at oesophagoscopy. In $60^{\circ}$, the proximal edge of the tumour in the lumen of the oesophagus was less than $30 \mathrm{~cm}$. from the upper alveolus. In those tumours that were resected the proximal edge of $36 \%$ lay less than $30 \mathrm{~cm}$. from the upper alveolus. This rather arbitrary division into high

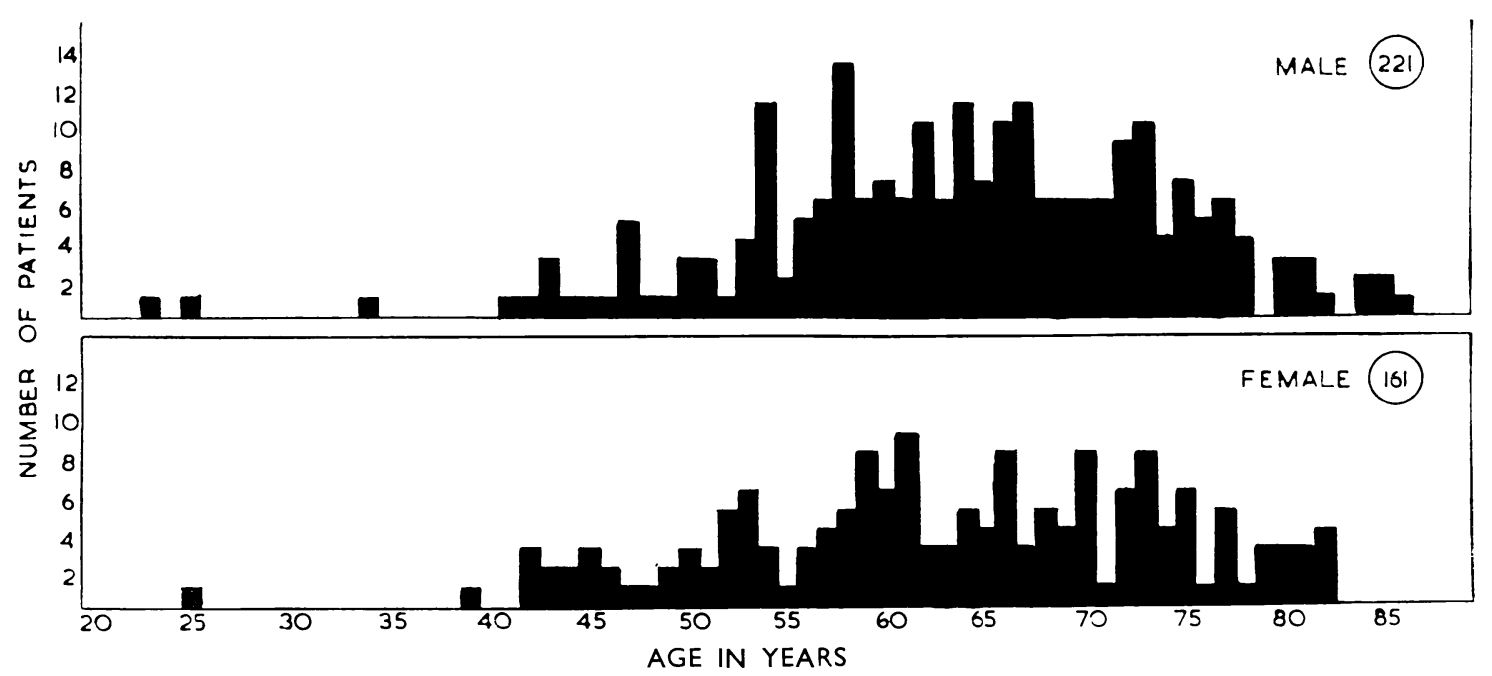

FIG. 4.-Age and sex distribution of patients with squamous carcinoma of the oesophagus. 


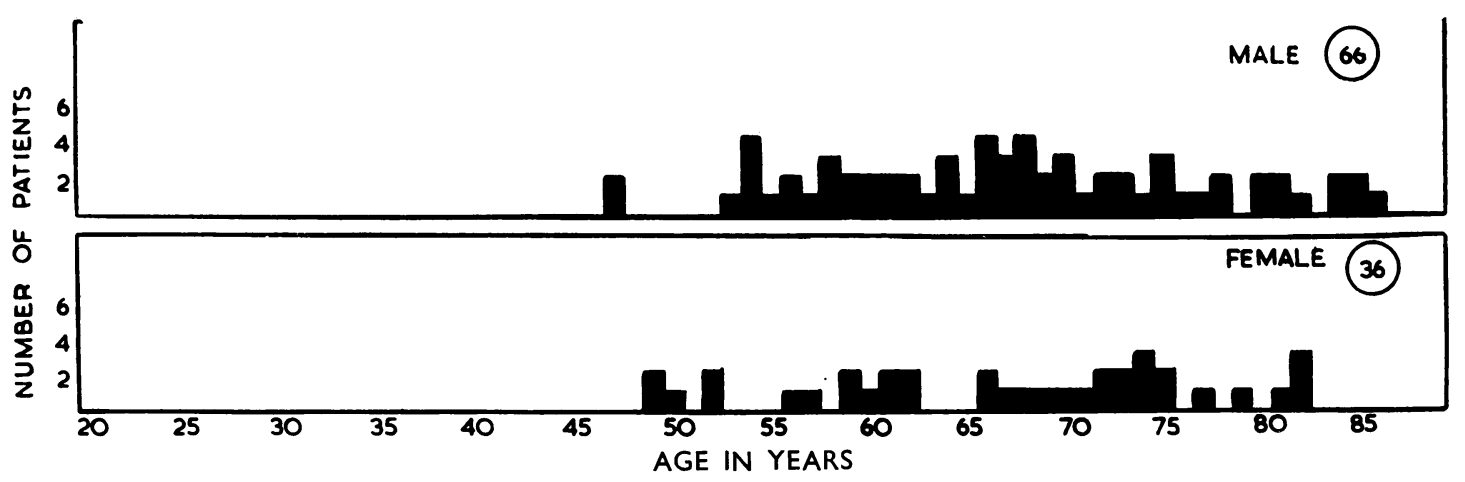

FIG. 5.-Age and sex distribution of patients with squamous carcinoma of the oesophagus not submitted to operation.

and low oesophageal tumours serves its purpose in demonstrating the lower incidence of resectability in the more proximal tumours. The anatomical explanation for this may be adequate; the proximal half of the oesophagus is in such close contact with the trachea and bronchi that early invasion of these is likely.

All these patients gave a history of dysphagia. In two women this history of dysphagia was unusual. One had all the clinical, radiographic, and endoscopic features of cardiospasm for nine years when she was originally investigated. A single dilatation restored swallowing so nearly to normal that she did not attend again. Three years later she was again referred for investigation, this time because of hoarseness. She was shown to have a right recurrent laryngeal nerve palsy and a squamous carcinoma in the middle third of the oesophagus. Dysphagia had not recurred after the single dilatation of the cardia three years before. The second woman, with an unusual story, presented at the age of 74 with increasing dysphagia for three months and respiratory symptoms. She was shown to have a tracheooesophageal fistula through squamous carcinoma, and she died from aspiration pneumonia ; she had been irradiated elsewhere 14 years before for a

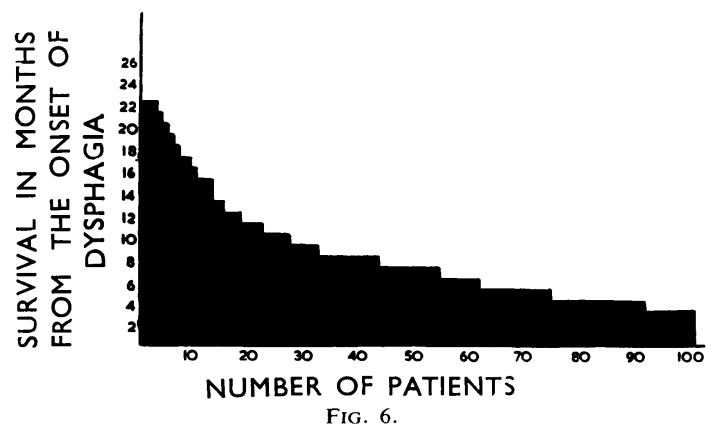

histologically proven squamous carcinoma of the oesophagus at the level of the aortic arch; irradiation had partially relieved her dysphagia at that time and she had lived on liquids and purées for 14 years.

Since no operation was undertaken, the duration of dysphagia in this group is synonymous with survival from the onset of dysphagia (Fig. 6). Nine patients died within three months of the onset of dysphagia. Omitting the two patients with the unusual histories, the three longest survivors lived 22 months from the onset of dysphagia, one without any treatment and two with radiotherapy. Again omitting the two women mentioned above, the average survival from the onset of dysphagia in 100 patients with squamous carcinoma of the oesophagus not submitted to operation was eight months. Survival is a little longer than in those with an adenocarcinoma not submitted to operation. The average duration of dysphagia before admission to hospital was just over four months, and this is not significantly different from that in the other groups discussed. Dysphagia was the first symptom recognized in all these patients; in 20 hiccup was another early symptom, and in 30 pain was a major complaint. Those with tracheal or bronchial invasion and those with total dysphagia, altogether 56 patients, had important respiratory symptoms.

The reasons for not undertaking resections in these patients were as follows:

Thirty-four patients were shown histologically to have invasion of the trachea or one or other main bronchus. The trachea was invaded in 15 , the left stem bronchus in 12 , the right in six, and both stem bronchi in one. One patient with tracheal invasion had, in addition, cervical glandular metastases. 
Twenty-six patients had histologically proven cervical glandular metastases, 15 on the left side, seven on the right, and in four there were glands on both sides of the neck. Two of these patients had an additional contraindication, namely, tracheal invasion in one and interruption of the right recurrent laryngeal nerve in the other.

Seven patients had a recurrent laryngeal nerve palsy (five left and two right); only one of the patients with a right recurrent palsy had right cervical glandular metastases.

Six patients had clinically palpable hepatic metastases, subsequently confirmed histologically after necropsy.

Three patients had histologically proven cutaneous metastases.

Twenty-six patients were not submitted to operation because of non-metastatic contraindications. In six, resection was not undertaken because of their poor general condition; the respiratory reserve in a further six was so limited that they were thought unable to tolerate thoracolaparotomy; and in 14 resection was not undertaken because of their advanced years.

Two patients declined treatment.

This makes a total of 102 patients (two having two distinct contraindications) who were not submitted to operation.

Seventeen patients were treated by radiotherapy. From Fig. 7 it will be seen that nearly all the longer survivors from the time of admission to hospital were irradiated. In nine of these 17 dysphagia was unaffected by irradiation, and these nine patients deteriorated rapidly. In the other eight dysphagia was relieved, three surviving irradiation by more than a year, but none surviving two years. In four a radium bougie was used; all these were treated more than 10 years ago; none of them survived treatment more than five months, and two died from massive haemorrhage.

In 35 patients dysphagia was partially relieved by intubation with a Simmonds' or Souttar's tube. Either of these tubes allows of the passage of only liquids, and there is consequently no indication for their insertion through the irresectable carcinoma of a patient able to swallow liquids without discomfort. Death in three of these patients was precipitated by perforation of the oesophagus at subsequent attempts at intubation, necessitated by blockage of a previous tube. Those intubated did not survive a significantly longer, or shorter, period than those whose dysphagia was not total and who were therefore not intubated. Three patients intubated were, in addition, irradiated.

Information regarding the mode of death in many who died at home is scanty, but, relying largely on the memory of the general practitioners concerned, a terminal respiratory illness was very common. Those patients with a fistula into the respiratory tract all died as a consequence of aspiration pneumonia, and nearly all these patients died in hospital. Seven patients, including two treated with radium bougies, died from massive haemorrhage.

Summary.-One hundred and two patients with squamous carcinoma of the oesophagus were not submitted to operation; $60 \%$ had high tumours,

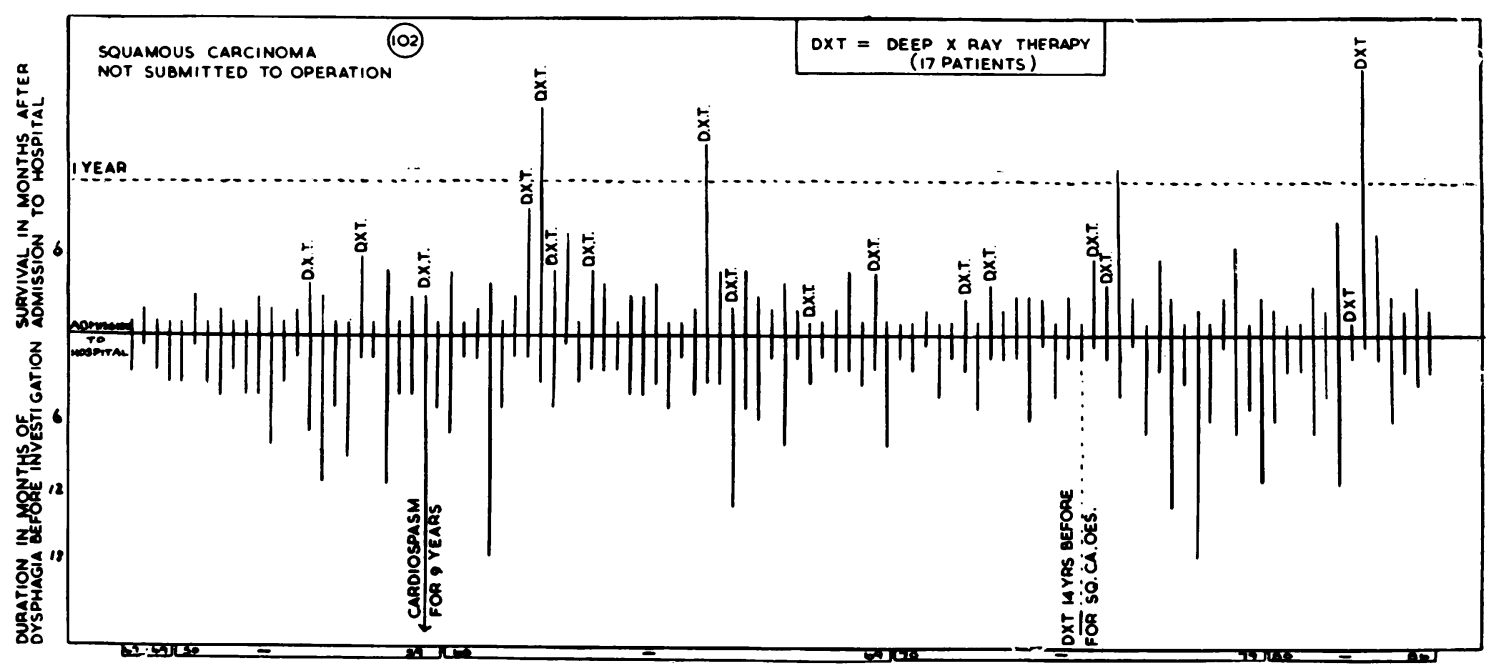

Fig. 7.-Each vertical line represents one patient, and these are arranged the youngest on the left and the oldest on the right. 


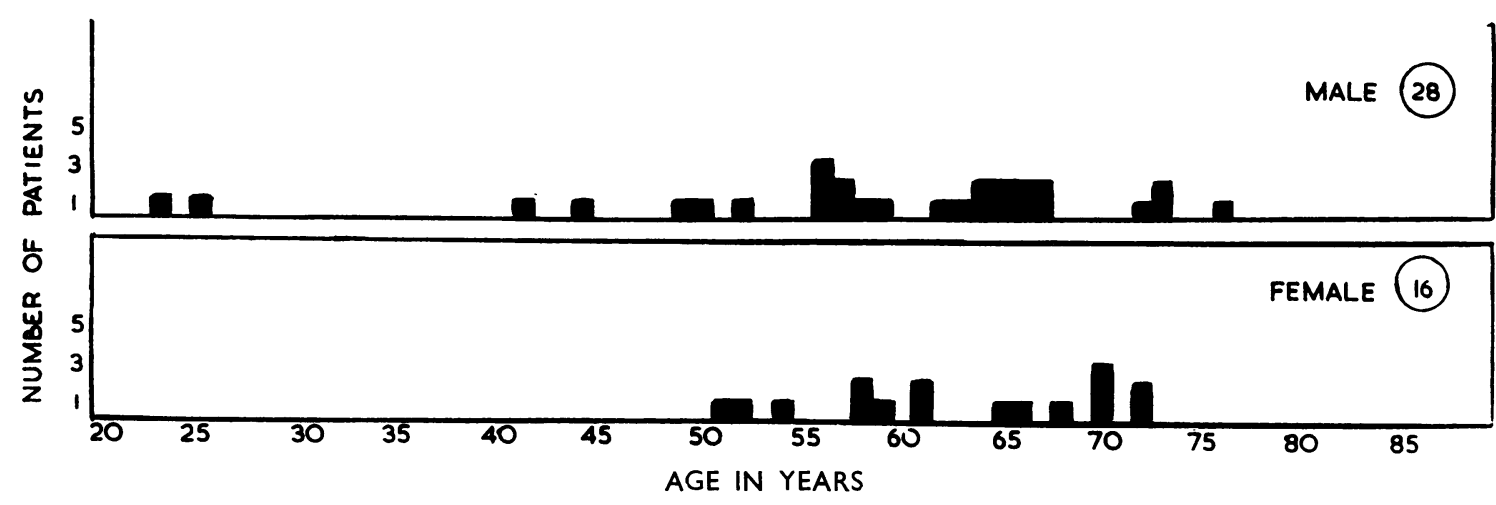

FIG. 8.

and invasion of the trachea or main bronchi was the commonest reason for not attempting resection. Radiotherapy temporarily relieved dysphagia in about half of those in whom this form of treatment was used. Average survival from the onset of dysphagia was eight months. A terminal respiratory illness was very common.

Squamous Carcinoma Found Irresectable at THORACO-LAPAROTOMY.-There were 44 patients submitted to thoraco-laparotomy with a view to resection (16 women and $28 \mathrm{men}$ ) in whom the tumour was found unsuitable for resection. The age and sex distribution of these patients are shown in Fig. 8. Two men in this group were unusually young, 23 and 25 years of age. Resection was not undertaken in 12 because of invasion of the aorta; in seven because of invasion of the lungs or bronchi; in six because of hepatic metastases; in four because of diffuse pleural metastases; in three because of cardiac invasion : and in a further three because of invasion of the posterior abdominal wall. In nine resection was abandoned because of what is loosely referred to in the operative notes as "mediastinal invasion."

In seven patients an attempt was made to re-establish swallowing by fashioning a bypass, using jejunum in three, stomach in three, and colon in one.

At operation three patients were found to have, in addition to the oesophageal tumour, a carcinoma of the stomach at the pyloric end: in all three the gastric tumour was an adenocarcinoma. Operation was precipitated in three patients by perforation of the oesophagus at oesophagoscopy. In these three an attempt was being made to intubate the tumour to promote hydration as a preliminary to resection. In one thoraco-laparotomy was undertaken in the presence of known cervical glandular metastases in an attempt to restore swallowing. In 10 the tumour was at the level of the aortic arch or higher and the approach was therefore from the right. In the remaining 34 a left thoraco-laparotomy was made. In 17 the proximal edge of the tumour, as seen endoscopically, was less than $30 \mathrm{~cm}$. from the upper alveolus, an incidence of " high" oesophageal tumours of $40 \%$, which is a little higher than that amongst the squamous tumours for which resection was completed.

Fourteen patients died as a direct consequence of operation, an operative mortality of $32 \%$ (Fig. 9). Two died from pulmonary embolism and seven from respiratory infection. Three patients for whom a bypass had been made died from disruption of the anastomosis. The remaining two died as a consequence of rupture of the oesophagus which became clinically obvious late in the first post-operative week but which had probably been initiated by the attempts at mobilizing the oesophagus.

Thirty patients survived operation. Swallowing had been restored in four of these by a bypass procedure. A further 10 were intubated with a Simmonds' or Souttar's tube and left hospital able to swallow liquids. Three patients treated about 10 years ago were treated with a radium bougie, and one, in addition to being intubated, was irradiated.

All these patients are dead. The duration of pre-operative dysphagia and the length of postoperative survival are shown in Fig. 9. The longest survivors include the four patients with the bypass procedure. Only one patient survived operation by more than a year. Survival from the onset of dysphagia is shown in Fig. 10 . Two patients survived only one month from the onset of 


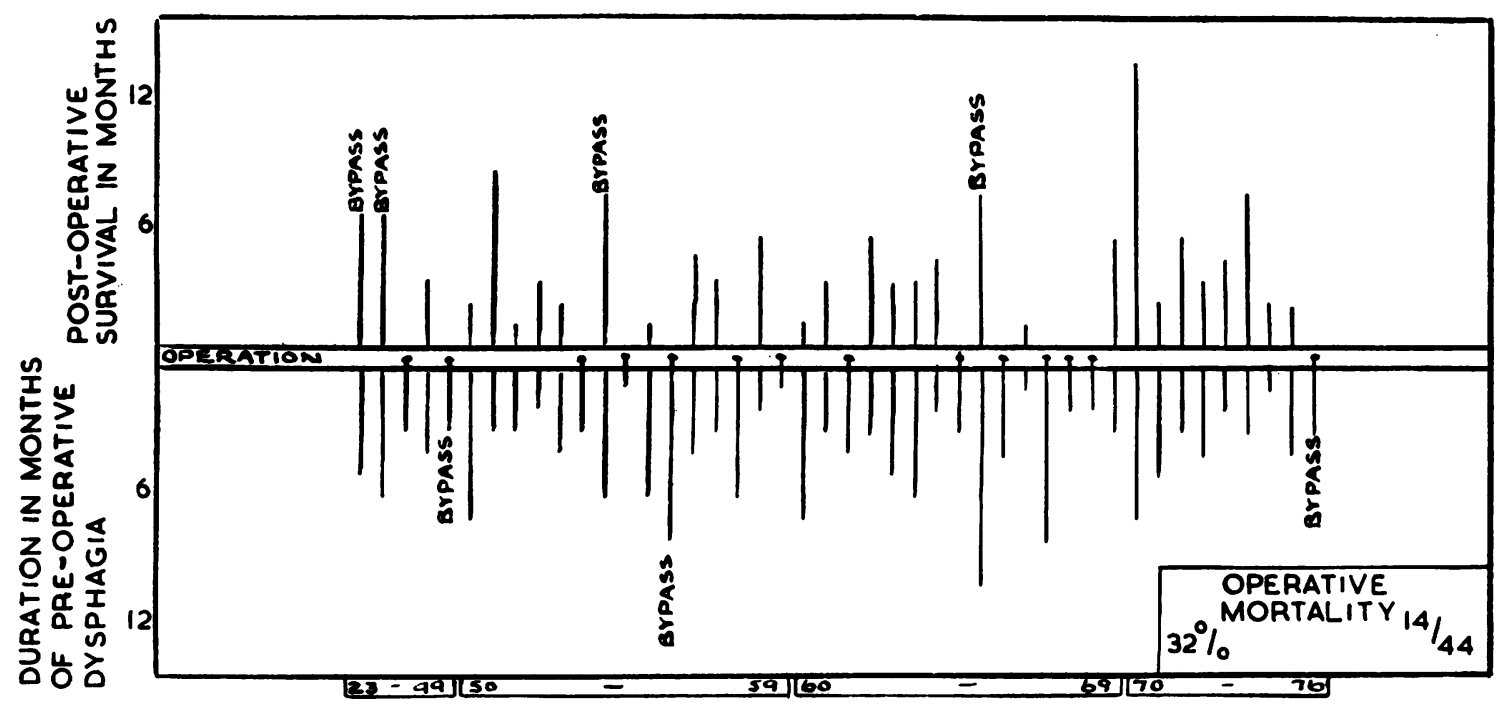

FIG. 9.-Squamous carcinoma found to be irresectable. Patients dying from operation are indicated by the dot in the transverse gap labelled operation .

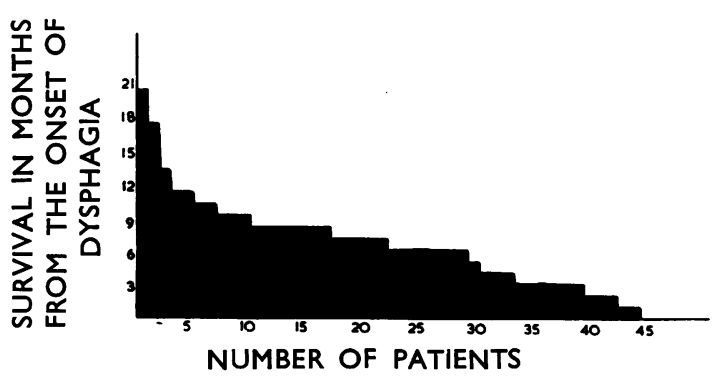

FIg. 10.

dysphagia and both died as a consequence of operation. The patient who survived more than a year following thoraco-laparotomy was also the longest survivor from the onset of dysphagia, 20 months. The average survival from the onset of dysphagia was just under seven months and the average duration of dysphagia before operation was four months.

Dysphagia was the first symptom in all these patients. The level at which the patient appreciated the obstruction had little bearing on the site of the tumour, the patients appreciating obstruction at one of three levels-in the suprasternal notch, at midsternal level, or in the epigastrium. Obstruction was usually appreciated at a level proximal to the tumour, and occasionally at a level distal to the tumour. In addition to dysphagia, pain, usually related to swallowing but sometimes not, and experienced in the epigastrium, in the suprasternal notch, at midsternal level or between the scapulae, was an obtrusive symptom in nine. In two patients hiccup was an annoying symptom which developed at the onset of dysphagia but which had disappeared by the time the patients were admitted for investigation.

Summary.-Forty-four patients with squamous carcinoma of the oesophagus were found at thoraco-laparotomy to have tumours unsuitable for resection. The operative mortality was $32 \%$ and average survival from the onset of dysphagia in these patients was a little less than in the group not submitted to operation.

Squamous Carcinoma Resected. - Two hundred and thirty-six patients with squamous carcinoma of the oesophagus were submitted to resection, 109 women and 127 men. The age incidence in the two sexes is shown in Fig. 11. The youngest was 25 years of age and the oldest 82. The average duration of pre-operative dysphagia was four to five months but nearer four months in the men and five months in the women. There is no significant difference in the average duration of dysphagia before resection in comparison with the duration of dysphagia in those patients who presented with metastases or in those with tumours found irresectable at thoracotomy, or in patients with adenocarcinoma. Symptoms in this group were no different from those with unresected tumours.

The Operation.-After resection of the tumour an oesophago-gastric anastomosis was made in 219 


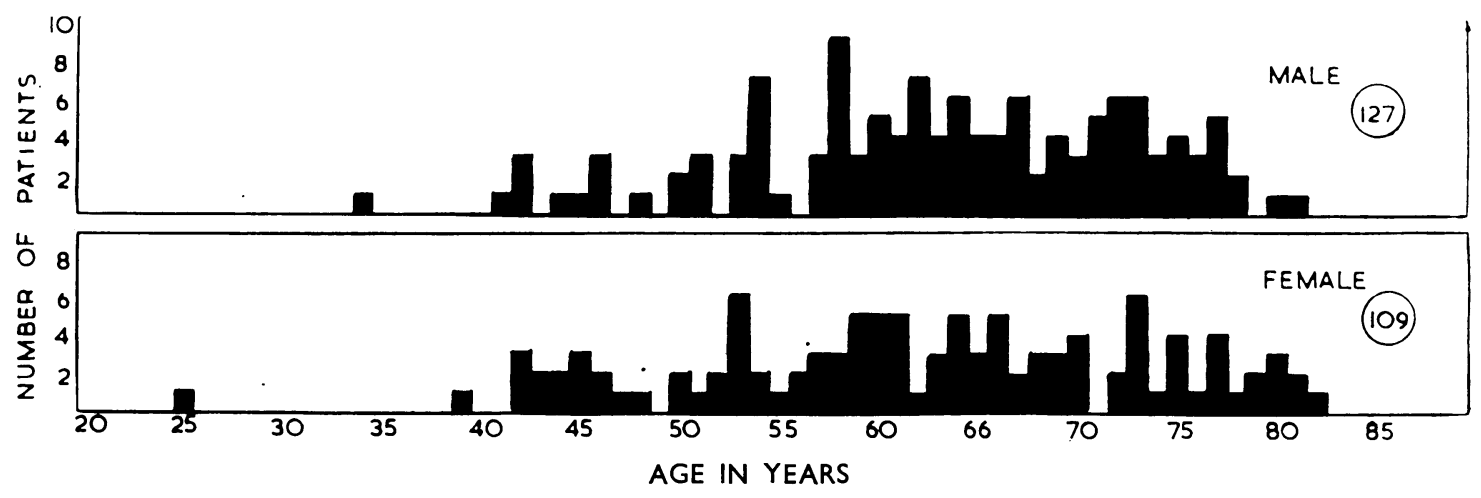

FIG. 11.

patients and an oesophago-jejunal anastomosis in 16. In one patient continuity was re-established with a plastic tube in the mediastinum. She died from metastases eight months after resection. In 44 patients the tumour was approached through a right thoraco-laparotomy, or through a right thoracotomy after preliminary mobilization of the stomach through a left paramedian laparotomy. In 192 the approach was from the left. In 53 in whom a left thoraco-laparotomy was used the anastomosis was made above the level of the aortic arch, the stomach lying lateral to the arch. The patients in whom a right approach was used and those in whom a supra-aortic anastomosis was made on the left are regarded as having a high oesophageal tumour, and these constitute $36 \%$ of the squamous tumours resected. If the somewhat arbitrary level of the proximal limit of the tumour as seen endoscopically is used to differentiate high from low tumours, and this level is fixed at $30 \mathrm{~cm}$., then again in this series $36 \%$ of the tumours are high. While these percentages are identical, there is a little difference in the representation of patients in the two groups. This difference is less than $5 \%$ and is explicable. The level of $30 \mathrm{~cm}$. is not correlated with the height of the patient or the position of the aortic arch; there is some variation in factors influencing the decision to make an anastomosis below the arch, both from surgeon to surgeon and at different times, and where the tumours are mobile the proximal edge can be made to move 3 or $4 \mathrm{~cm}$. so that the level can be varied by pressure from the endoscope. The endoscopic evidence of the proximal limit of the tumour has nevertheless been found more dependable than the radiographic evidence. Only those tumours obviously at or above the level of the aortic arch were approached from the right.
Operative Mortality.-Eighty patients died as a direct consequence of operation; 40 were men and 40 were women, and the operative mortality was $34 \%$. The operative mortality amongst men (32\%) was lower than amongst women $(37 \%)$. Most of the operative deaths amongst the women were in those over 65 years of age (Fig. 12), of whom $50 \%$ died from operation. Amongst the men there is a fairly even scatter of operative deaths under 70 years of age, but over 70 the operative mortality was $50 \%$ (Fig. 13). Of the 44 patients in whom the approach was from the right, $15(34 \%)$ died from operation, an operative mortality for this approach corresponding with that of all squamous carcinomas which were resected. Of the 53 patients in whom a supra-aortic anastomosis was made, $23(43 \%)$ died from operation. The operative mortality for patients with "high" tumours-a combination of those approached from the right and those in whom the anastomosis was above the aortic arch -is therefore $39 \%$ against an operative mortality of $30 \%$ amongst the resections for "low" squamous tumours, since of 139 patients in whom the anastomosis was made at or below the level of the lower border of the aortic arch, 42 died. Amongst the resections for adenocarcinoma none of the few patients whose tumour was approached from the right or in whom the anastomosis was made above the level of the aortic arch died from operation.

Seventeen operative deaths, nine of which occurred in women, were a consequence of leakage from the anastomosis, and a further five operative deaths were from haemorrhage, a consequence of erosion of the aorta, a late sequel, it is believed, to leakage from the anastomosis. Three patients approached from the right and six patients in whom a supra-aortic anastomosis were 


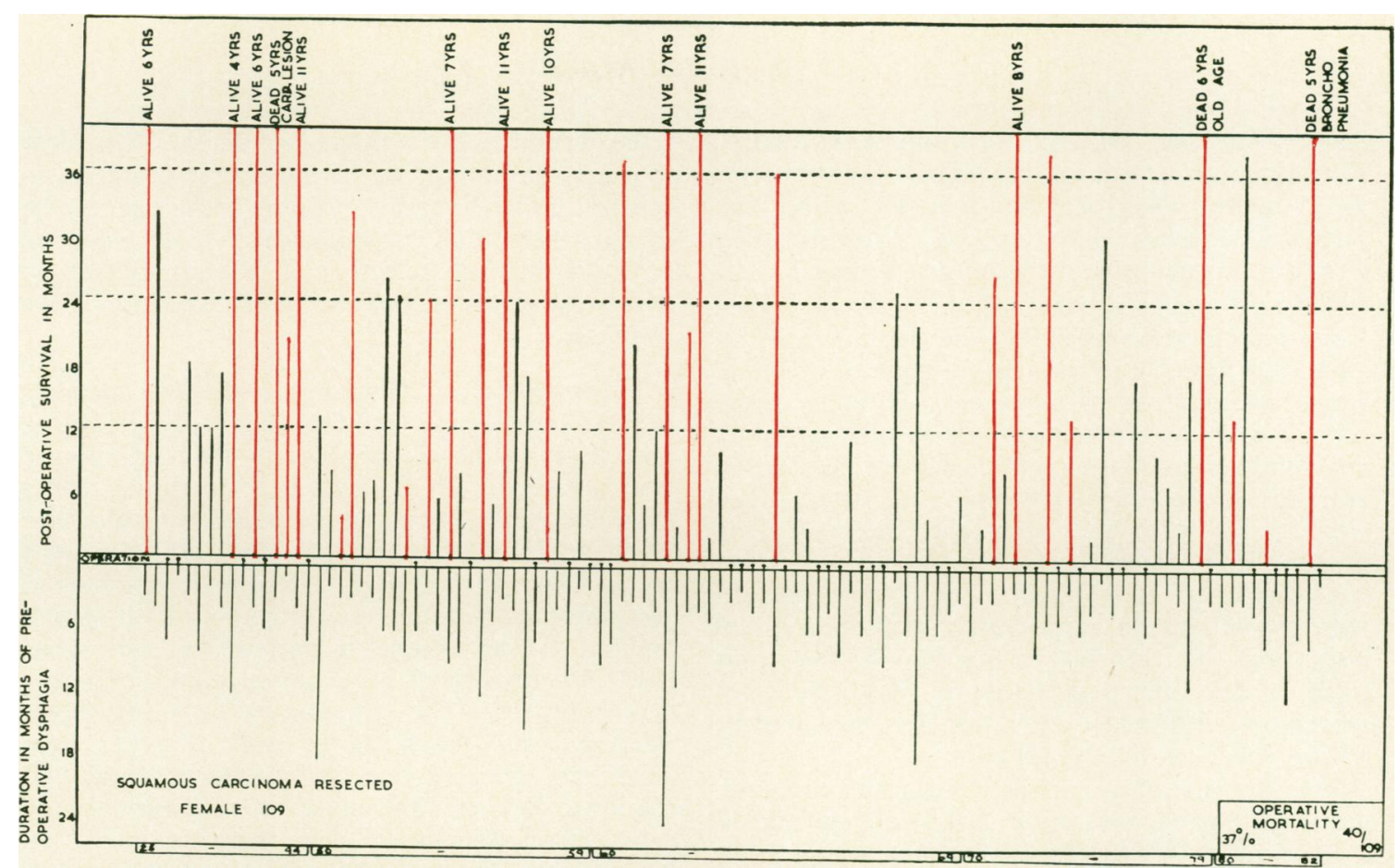

FIG. 12.-Each vertical line represents a patient, and these are arranged youngest on the left, oldest on the right. Patients dying from operation are indicated by the dot in the transverse gap labelled operation. The red lines represent patients who are alive at the present time or who have died more than five years after operation but not from metastases. The vertical black lines above the operative gap represent patients who have died from metastases or who have died less than four years after operation from causes other than metastases.

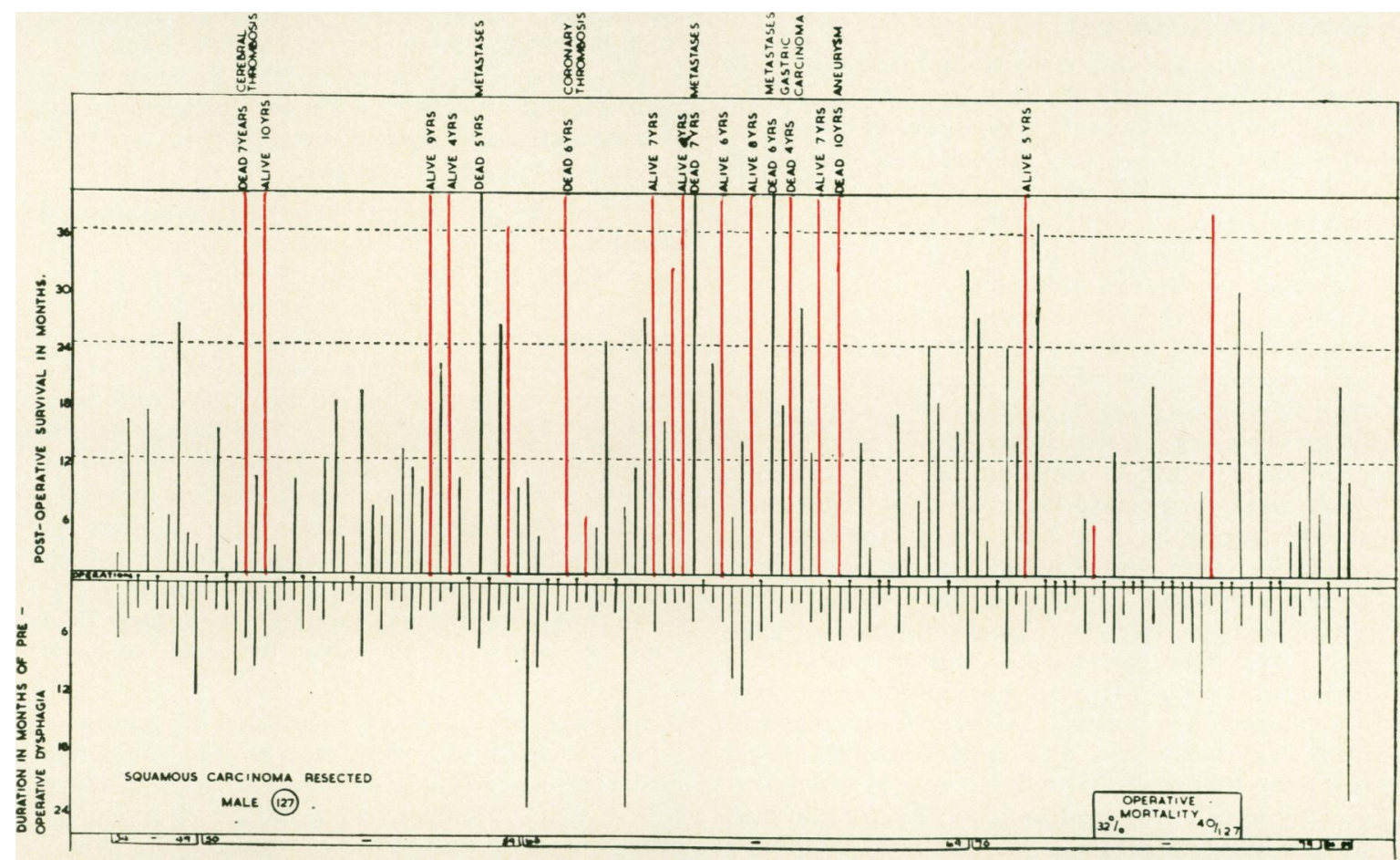

FIG. 13.-Each vertical line represents a patient, and these are arranged youngest on the left, oldest on the right. Patients dying from operation are indicated by the dot in the transverse gap labelled operation. The red lines represent patients who are alive at the present time or who have died more than five years after operation but not from metastases. The vertical black lines above the operative gap represent patients who have died from metastases or who have died less than four years after operation from causes other than metastases. 
made died from leakage from the anastomosis. Fifty-three per cent. of inadequate anastomoses were made, therefore, in patients with high tumours, whereas high tumours comprised only $36 \%$ of the squamous carcinomata resected.

Thirteen patients, eight of them women, died from pulmonary embolism, and 19, 13 of them men, died most directly from pulmonary infection. Three patients, two of them approached from the right, died from empyema thoracis with an intact anastomosis and one patient died from necrotizing enteritis on the fourth post-operative day. Three women died with an uncontrolled leak of chyle into the right pleural space ; pulmonary infection contributed to their death. In all three deaths with chylothorax a supra-aortic anastomosis had been made, but this relationship may be fortuitous since, of five other chylothoraces which were not associated with death, only one was in a patient with a supra-aortic anastomosis.

Five patients, three of them with high tumours, remained hypotensive after operation and failed to respond to resuscitative measures, dying more or less directly from surgical " shock" during the first three post-operative days. Two women died from reactionary haemorrhage during the first eight hours after operation from sources not determined at necropsy.

Three men and one woman died from coronary artery disease during the first four post-operative days; three of them had post-mortem evidence of recent coronary artery occlusion and the fourth had grossly diseased coronary arteries and died in cardiac failure. Two men died with renal insufficiency, one being found at necropsy to have a chronic destructive inflammatory lesion in both kidneys and the other acute tubular necrosis, possibly related to a period of hypotension in the early post-operative phase. One woman and one man died during the first two post-operative days from primary respiratory insufficiency, with respiratory infection contributing to their death. They had gross emphysema and, in retrospect, should probably not have been subjected to resection. One woman died from hepatic failure consequent upon ligation of the hepatic artery which was involved in a mass of tumour. One man died four hours after operation with an unrecognized right tension pneumothorax ; in him oesophagectomy through a left thoraco-laparotomy had been associated with transmediastinal resection of part of the right lower lobe which was directly invaded by the tumour. One woman died with a left hemiplegia from cerebral thrombosis and another died with severe parotitis as the only lesion found to account for her death. It is clear that a large proportion of the operative deaths in this series are the result of faulty technique. Some of the others are a consequence of faulty selection of patients, but selection is always biased in favour of resection because of the need to relieve dysphagia.

Metastases at Operation.-In 145 patients (61\%) histological confirmation of glandular metastases in the abdomen or in the mediastinum was obtained. In 53 evidence of spread other than glandular invasion was found, but only four of these 53 did not have, in addition, glandular metastases. In all, $63 \%$ of patients in whom a squamous carcinoma was resected had spread beyond the oesophagus in comparison with more than $70 \%$ of patients in whom an adenocarcinoma was resected. The commonest sites of direct invasion were the posterior wall of either stem bronchus, the posterior wall of the trachea, and the lower lobe of either lung, more commonly the right, in relation to the pulmonary ligament. In 29 patients tracheal, bronchial, or pulmonary invasion was found. Resection was undertaken in nine found at operation to have hepatic metastases, and two of these did not have glandular metastases. These nine with hepatic metastases were relatively young and had severe dysphagia. Tumour was left adherent to the aorta in seven patients, and elsewhere in the mediastinum in six. Diffuse peritoneal spread was found in one, and a renal metastasis in another.

Part of the pericardium was resected because of invasion by tumour in 17 patients. Resection was undertaken in three with histologically proven cervical glandular metastases and in a fourth with a left recurrent laryngeal nerve palsy presumed to be a consequence of mediastinal invasion. These four were young, had severe dysphagia, and were in good general condition. Resection in the presence of known cervical glandular metastases enabled a 39-year-old woman to swallow normally for three years after resection while she cared for a young family. Of the other three in whom resection was undertaken despite known metastases, one with cervical glandular metastases died from operation and the other two each survived a year.

Whereas $63 \%$ of all patients in whom a squamous tumour was resected had operative evidence of metastases or invasion, $73 \%$ of those with " high" tumours had evidence of spread at operation, and only $55 \%$ of those with "low" tumours had spread at operation. Resection was 
undertaken in 87 patients (37\%) in the absence of histological evidence of dissemination at the time of operation, and, of these, 25 died as a consequence of operation. One hundred and fifty-six patients survived operation, 62 of them without histologically confirmed spread. Twenty-three of these 62 later died from metastases, 10 died from non-metastatic causes, and 29 are alive.

Operation in Unusual Circumstances.-Four patients in whom resection was undertaken were known to have two foci of squamous carcinoma in the oesophagus with normal oesophageal mucosa between them. Examination of the resected specimen showed submucous lymphatic extension of the primary tumour and the second malignant mucosal ulcer was therefore a metastasis. Two of these patients died from operation and two died from metastases during the second post-operative year. Resection was undertaken in two patients in whom dysphagia had been relieved for a short period by radiotherapy, only to recur. Three men gave a long history of intermittent dysphagia with a change in symptoms precipitating their admission to hospital. These three probably had benign, peptic strictures of the oesophagus. All survived operation, two died from metastases within a year of operation, one survived 10 years and then died after resection of an abdominal aortic aneurysm. A woman gave a history of 17 years of intermittent dysphagia; she had a web at the crico-pharyngeus and a squamous carcinoma in the distal oesophagus, and she was anaemic; she is probably an example of sideropenic dysphagia complicated by carcinoma other than in the hypopharynx. In three in whom resection was undertaken an additional malignant tumour was found: an adenocarcinoma of the distal part of the stomach in two and in the third the microscopic glandular and splenic changes of chronic lymphatic leukaemia. In two patients the left recurrent laryngeal nerve was sacrificed at operation to clear glandular metastases.

Non-Fatal Complications.-Five of the eight patients whose convalescence was complicated by chylothorax survived. The chylous effusion was on the right side in all these, and they were treated by intercostal intubation with drainage into a water-seal. Two patients survived leakage from the alimentary tract into the left pleura. One, in whom a leak was demonstrated on the ninth post-operative day, was re-explored and found to have a perforation of the intrathoracic gastric tube $5 \mathrm{~cm}$. distal to the oesophago-gastrostomy. This was regarded as a perforated peptic ulcer, but may have been the result of trauma to or ischaemia of the gastric wall. The perforation was closed and the patient's recovery thereafter was uneventful. The other was found at re-exploration on the seventh day to have a disrupted oesophago-gastric anastomosis; the proximal oesophageal stump was delivered on to the surface of the skin in the left side of the neck, just above the clavicle, and the gastric stump was exteriorized at the left costal margin and used as a gastrostomy for purposes of feeding. She was left with these fistulae while the left empyema was managed and she improved over several months. Alimentary continuity was then re-established through the anterior mediastinum using jejunum, and she is well two years after completion of these surgical procedures.

The Fate of the Survivors After Operation -One hundred and fifty-six patients survived operation, 62 of these not having been found at operation to have histologically demonstrable evidence of dissemination. One hundred and eight patients, 67 men and 41 women, died subsequently from metastases. Ten have died from nonmetastatic causes, six of them more than five years after resection. Thirty-eight patients, 24 women and 14 men, are alive.

Of the 108 patients who died from metastases after resection, 59 died during the first year, 33 men and 26 women (Figs. 12 and 13). During the second year after resection a further 34 ( 24 men and 10 women) died from metastases, and during the third year after resection 11 patients (seven men and four women) died from metastases. During the fourth year after resection one woman died from metastases and three men died five, six, and seven years after resection respectively, also with metastases. Of those who died from metastases, eight who died within a year of operation, seven who died in the second year after operation, seven who died in the third year after operation, and one dying seven years after operation had not been shown to have metastases at the time of operation. Therefore, 23 of the 62 survivors of operation who had not been found to have evidence of spread at operation died from metastases.

A further 48 patients need to be accounted for. Five are alive less than a year after resection, and two of these had glandular metastases at operation while three had no evidence of spread. Five have survived more than a year after resection, but not more than two years ; two of these had metastases at operation and three did not. Five have survived more than two years after operation but 
have not yet survived three years; only one of these five had glandular invasion at operation, the other four not being shown at operation to have evidence of spread. Four are alive more than three and less than four years after resection, and none of these had glandular metastases at operation. Nineteen patients are alive more than four years after resection. Of these 19 , two are alive in the fourth post-operative year, one in the fifth, three in the sixth, four in the seventh, two in the eighth, two in the ninth, two in the tenth, and three in the eleventh post-operative years. Four patients surviving more than four years after resection had evidence of glandular metastases at operation, and one of these is alive seven and another nine years after resection.

Thirty-eight of the 48 patients previously unaccounted for are alive; the remaining 10 have died. Three women have died from nonmetastatic causes more than five years after operation: one from bronchopneumonia at the age of 86 , having survived resection by five years, one from old age at 86 , having survived resection nearly seven years, and one from a cardiac lesion at 50 , having survived resection by five years. Four men have died from non-metastatic causes more than four years after resection. One died seven years after resection at the age of 58 from cerebral thrombosis; one died six years after resection at 67 from coronary thrombosis; one died nearly five years after resection from massive bleeding from an adenocarcinoma of the stomach, resection having been for a squamous carcinoma of the oesophagus; and one died 10 years after resection as a consequence of an operation for an aneurysm of the abdominal aorta.

Three patients have died from non-metastatic causes less than four years after resection. A woman died from ruptured diverticulitis of the colon in the second year after resection; a man died from an adenocarcinoma of the rectum in the third year after resection; and a man died from bronchial carcinoma in the fourth year after resection. This last-mentioned man presented two years after resection with a ruptured aneurysm of the abdominal aorta distal to the renal arteries; this aneurysm was resected and replaced with a Dacron graft; he recovered rapidly from this procedure; no intra-abdominal metastases had been found at laparotomy. Two years later he presented with haemoptysis and was shown to have a squamous carcinoma in the right upper bronchus. His original oesophageal tumour had been a squamous carcinoma, but without glandular metastases. Because he was then 75 and with a poor respiratory reserve, he was not submitted to resection of the bronchial tumour; he died seven months after a course of radiotherapy. At necropsy the bronchial tumour was barely recognizable, having been satisfactorily shrunk by radiotherapy, and there were no evidences of spread in the mediastinum; the oesophago-gastrostomy looked normal; he died with severe coronary arteriosclerosis. He is the second man in this series to develop an abdominal aneurysm after oesophago-gastrectomy. The other died 10 years after oesophago-gastrectomy and 10 days after resection of an abdominal aneurysm, continuity again being established with Dacron. He died from respiratory infection, and at necropsy there was no evidence of metastases from the oesophageal carcinoma. The Dacron graft was patent and cleanly lined by a smooth, polished membrane, presumably composed of platelets and fibrin.

Of 236 patients submitted to resection and of 156 who survived operation, 17 are alive at the present time and more than five years after operation. Six other patients have died more than five years after resection from non-metastatic causes. Of the 236 resections, 128 were undertaken more than five years ago, and of these 128 patients, 37 died from operation. Only 91 patients have had the opportunity of surviving five years. If the five-year survival rate is calculated to include only those at present alive, then $13 \%$ of those submitted to operation, or $19 \%$ of those who survived operation, are survivors for five years. But survival from oesophago-gastrectomy does not confer immortality, and it is justifiable to include those now dead, but alive at five years, provided they did not die from metastases. The five-year survival rate is therefore $18 \%$, calculated against the number submitted to operation, and $25 \%$, calculated against those surviving operation. In all but one of the 23 patients alive at the end of five years the distal part of the stomach was used to re-establish continuity after resection of the tumour ; in one jejunum was used. Approximately one anastomosis in every 13 was made using jejunum.

ReCURRence of Dysphagia.-Dysphagia recurred before death in 33 of the 108 patients dying with metastases, and in 32 of these evidence of glandular invasion had been found at operation. In 14 of those dying with recurrence of dysphagia the original tumour had been high, and in 19 low. Fifteen died with recurrence of dysphagia during the first year after resection: 
eight of these had had high tumours, seven low, one of these seven without glandular metastases. Fourteen died with recurrence of dysphagia during the second year after resection, four with high and 10 with low tumours originally; and four, two of each, died during the third year after resection. In three dysphagia recurred nearly a year before death, and was treated by intubation of the recurrence at the anastomosis; in most of the remainder dysphagia recurred within two months of death and was only one of several manifestations of recurrence or spread in these patients. Malignant dysphagia recurred, therefore, in $21 \%$ of those surviving operation.

In three patients still alive and believed not to have metastases the oesophago-gastric stoma has required dilatation because of a fibrous stricture, probably of peptic origin. A fourth patient recorded earlier as dying six years after resection with metastases developed a peptic stricture at the oesophago-gastrostomy within a year of resection. $\mathrm{He}$ learned to use a gum-elastic bougie and maintained adequate swallowing with this for five years. A retired railwayman, he was granted free passage on the railways, and made the journey regularly from his distant home to Edinburgh, entertaining his fellow passengers by his demonstration of "sword-swallowing." He then began to feel less well, and asked that his stricture be dealt with surgically. The stricture was resected, and at this operation glandular metastases were found; he died some weeks later from urinary infection, but with fairly extensive metastases.

Fate of Those with High Tumours.-Ninetyseven patients were found to have "high" tumours, and 38 (39\%) of these died from operation. During the first year after operation 26 from whom high tumours had been resected died from metastases, eight of these with recurrence of dysphagia. During the second year after resection 11 died with metastases, four of them with recurrence of dysphagia; and during the third year after resection four died from metastases, two with recurrence of dysphagia. One died from metastases six years and one seven years after resection. Sixteen are alive, eight of them more than five years after resection, and three of these eight had glandular metastases at operation. Two of these are worthy of more detailed record.

One, a man who is alive six years after resection, was submitted at the age of 64 to resection of a squamous carcinoma, $23 \mathrm{~cm}$. from the upper alveolus, through a right approach; glands were invaded. A year later he was readmitted seriously ill with a mass of glands in the left side of the neck, shown histologically to contain squamous carcinoma, and with stridor, the consequence of squamous carcinoma invading the posterior wall of the trachea and left main bronchus at the main carina. To improve his airway a considerable quantity of tumour was resected with biopsy forceps through the bronchoscope, and he was irradiated. He is well, at the age of 70 , six years after resection and five years after irradiation, without clinical evidence of metastases, swallowing normally, and with normal bronchoscopic appearances. The second patient of particular interest in this group of longsurviving high tumours was the youngest submitted to resection, a woman, then unmarried, 25 years of age, with a squamous carcinoma at 25 $\mathrm{cm}$. from the upper alveolus. A supra-aortic anastomosis was made from the left; glands were not invaded; and she is alive, married now and with a family, six years after resection. Of the eight patients alive less than five years after resection of a high tumour, five had glandular metastases at operation and three did not. One of these last-mentioned was the woman mentioned above who survived leakage from the anastomosis, exteriorization of oesophagus and stomach and ultimate restoration of continuity.

Relative to their representation in the series of squamous carcinomas as a whole, more patients with high tumours died from operation and during the first year after operation from metastases than did patients with low tumours. Thereafter the survival rate of high and low tumours is the same. The higher death rate from metastases in the first year after operation in those with high tumours is probably a corollary of the higher incidence of metastases at operation in those with high tumours.

Incidence of Effective Palliation.-The palliative value of resection in those patients dying from metastases must be assessed against the average survival time of those patients with squamous carcinoma of the oesophagus not submitted to operation. In the group of 100 typical examples of squamous carcinoma not submitted to operation it has been shown earlier that the average duration of dysphagia before admission to hospital was four months, and that average survival from the onset of dysphagia was eight months. Eighty patients in whom a squamous carcinoma was resected died from operation; in these the average duration of dysphagia before operation was four months (Fig. 14a), and, since they died from operation, 


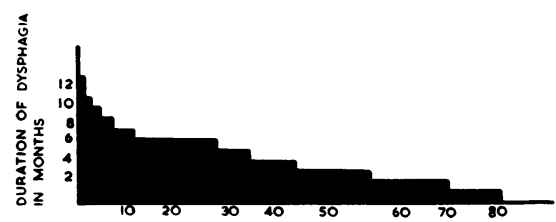

(a)

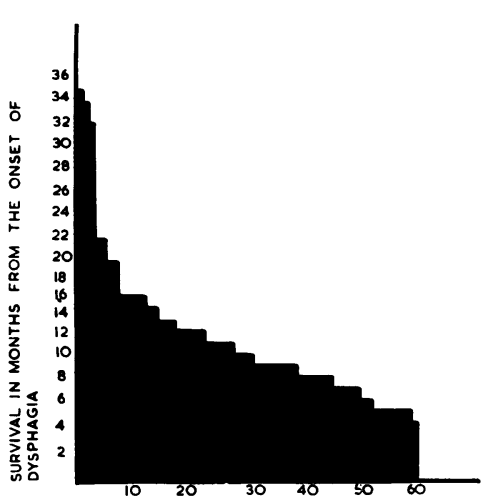

(b)

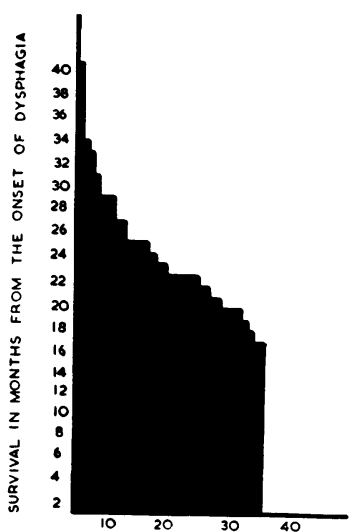

(c)

NUMBER OF PATIENTS

FIG. 14.

average survival from the onset of dysphagia was also four months. No palliative benefit accrued to them. Fifty-nine died from metastases during the first year after resection, and, of these 59, 15 died with recurrence of dysphagia. The average survival from the onset of dysphagia in this group of 59 was just over 12 months, the longest survival being 34 months and the shortest five months (Fig. 14b). The restoration of swallowing for even a limited period was probably regarded by these patients as valuable, but as regards duration of survival little palliative benefit can be claimed, especially since $25 \%$ died with recurrence of dysphagia. Resection in 139 patients $(80+59)$ can therefore be regarded as little more than an episode in the natural course of the disease, i.e., in $60 \%$ of resections undertaken the natural history of the disease was unaltered. Thirty-four patients died from metastases during the second year after resection, 14 of these 34 dying with recurrence of dysphagia. The average survival from the onset of dysphagia in this group of 34 was 23 months, the longest survival being 40 months and the shortest 15 months (Fig. 14c). Significant palliative benefit, i.e., increase in survival time from the onset of dysphagia, can be claimed for these patients, and for all those dying subsequently from metastases.

Summary. - Two hundred and thirty-six squamous tumours of the oesophagus were resected; $36 \%$ were high tumours. Thirty-four per cent. of patients died as a consequence of operation; $63 \%$ had evidence of spread at operation. Forty-six per cent. of those submitted to operation $(69 \%$ of those who survived operation) died from metastases, more than half of them during the first 12 months after resection.
Ten per cent. of those submitted to operation $(15 \%$ of those who survived operation) were alive five years after resection, although some of these have since died from non-metastatic causes. Three patients died more than five years after resection from metastases. Ten per cent. of those submitted to operation are alive less than five years after resection, or have died from non-o metastatic causes during the first five postoperative years.

\section{UNDIFFERENTIATED CARCINOMA OF THE Oesophagus}

Twenty-six patients investigated because of dysphagia were shown to have an oesophageal carcinoma histologically called undifferentiated. The sexes were equally represented. Thirteen of these patients had metastases when first seen, and one had coronary artery insufficiency of such severity that thoraco-laparotomy was precluded. The age incidence of the 14 patients not submitted to operation is shown in Fig. 15. The sexes in this group are again equally represented.

Not Resected.-Of the 13 patients with metastases, four had histologically proven spread to left cervical glands, two had tracheal and three left main bronchus invasion confirmed histologically, two had hepatic metastases palpable clinically and later demonstrated at necropsy, and two had diffuse pulmonary metastases also confirmed at necropsy. In nine of these 13 patients the proximal edge of the tumour was seen oesophagoscopically less than $30 \mathrm{~cm}$. from the upper alveolus.

Four of these 14 patients were treated by irradiation (Fig. 16). One, the man in whom 
thoraco-laparotomy was precluded because of coronary artery disease, is alive seven months after the onset of dysphagia and four months after completing radiotherapy. The longest survivor from the onset of dysphagia-28 months-was irradiated. The third patient irradiated had an unusually long history of dysphagia - three yearsbefore admission to hospital, but survived irradiation by only two months. The fourth patient irradiated died three months after admission to
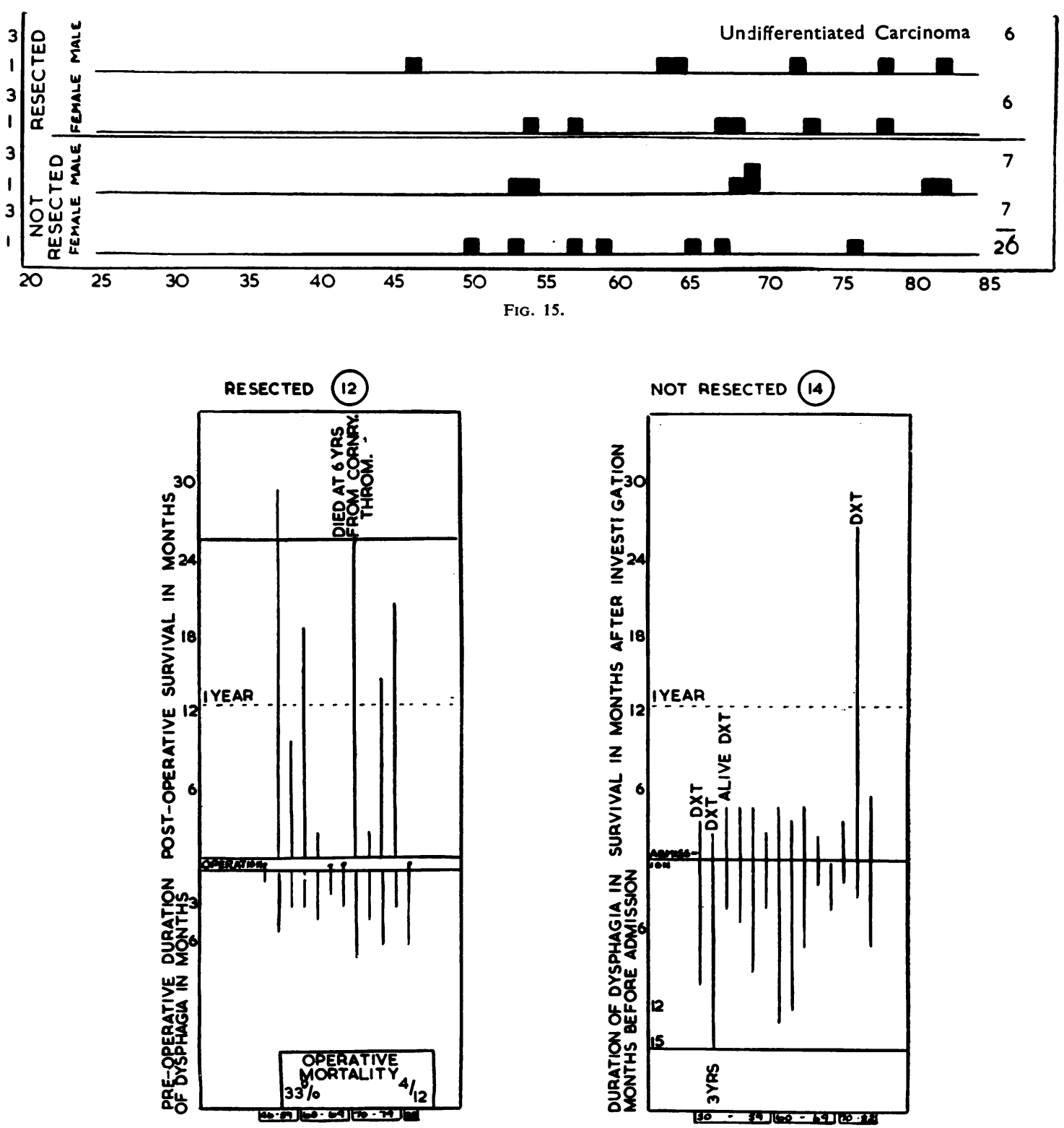

FIG. 16.-Each vertical line represents a patient. The patient represented by the shorter red line although alive has metastases at the present time. The longer red line represents a patient dying from a cause other than metastases six years after operation. DXT is an abbreviation for deep $x$-ray therapy. The patients are arranged the youngest on the left, oldest on the right. 
hospital. Three patients with total dysphagia were enabled to swallow liquids by the introduction of a Souttar's tube.

The average duration of dysphagia before admission to hospital in the 14 patients not submitted to resection was seven months; if the patient with the unusually long history of dysphagia is excluded, this figure is reduced to six months. The average survival from the onset of dysphagia in this group is 11 to 12 months.

RESECTED.-Twelve patients (six men and six women) with an undifferentiated oesophageal carcinoma were submitted to resection (see Fig. 16). The age incidence is shown in Fig. 15. In only three of these was the proximal edge of the tumour less than $30 \mathrm{~cm}$. from the upper alveolus. In 11 an oesophago-gastric anastomosis was made and in one an oesophago-jejunal anastomosis. Two of the oesophago-gastrostomies were supraaortic, and a third was made through a right thoraco-laparotomy. Four patients died from operation, an operative mortality of $33 \%$. One died from leakage from the anastomosis, one from haemorrhage, a consequence of erosion of the aorta, one from pulmonary infection, and one, in whom an extensive gastrectomy had been made, jejunum being used to restore continuity, died on the tenth day from peritonitis, a consequence of rupture of the duodenal stump. One patient died suddenly a month after leaving hospital, two months after resection. The cause of death is not known, but was not, so far as can be ascertained, from haemorrhage.

In 10 of the 12 patients glandular metastases were found at operation. The four operative deaths and the sudden death two months after resection were all in patients who had glandular metastases. Four patients died from metastases: one two months after resection, one nine months after resection, and two at 20 and 21 months respectively. These four patients had glandular invasion demonstrated at operation. A fifth patient died from metastases 29 months after resection, and in this patient no evidence of spread had been found at operation. One man with glandular invasion at operation is alive 14 months after resection but is ill with clinical evidence of hepatic metastases. One patient died from coronary thrombosis six years after resection. He is the second patient who did not have evidence of spread at operation. Dysphagia did not recur in any patient submitted to resection.

Excluding the patient dying from coronary thrombosis, and limiting the prognosis of the patient alive with hepatic metastases to three months, the average survival from the onset of dysphagia in those undergoing resection is 12 months, including those dying from operation, and this does not differ from the duration of survival in those not submitted to operation; excluding those dying from operation the average survival from the onset of dysphagia is 20 months. The average duration of dysphagia before resection was four months.

Thus, to summarize, 26 patients were found to have an undifferentiated carcinoma of the oesophagus. The sexes in this group were equally represented. Thirteen were not submitted to operation because of metastases and in these average survival from the onset of dysphagia was 11 to 12 months. Resection was undertaken in 12, in 10 with glandular metastases. One patient survived more than five years, four $(33 \%)$ died from operation, one is alive with metastases, and the remainder have died with metastases. The average estimated survival time from the onset of dysphagia in those dying from operation and metastases is no different from those not resected, but this average is heavily biased by the operative mortality. Resection is justified by three patients surviving more than 18 months and a fourth six years from a series of 12 .

\section{Adenocarcinoma in the Oesophagus and Proximal Stomach}

Of the 700 patients found to have a primary carcinoma of the proximal alimentary tract during investigation for dysphagia, 272 (39\%) were shown to have an adenocarcinoma. The age and sex distribution of these patients are shown in Fig. 17. The incidence amongst men is a little more than twice that amongst women. The tumour was visible oesophagoscopically in 180 and was not visible through the oesophagoscope in 92. Resection was undertaken in 170 patients $(62 \%)$, an incidence of resectability the same as that for squamous carcinoma of the oesophagus. Fortysix patients were submitted to thoraco-laparotomy with a view to resection but were found to have either an irresectable tumour or one from which dissemination was so gross that resection was unjustified because of the relative mildness of dysphagia. Operation was therefore frustrated in $17 \%$ of patients with adenocarcinoma in comparison with $12 \%$ of patients with squamous carcinoma. In 56 patients $(21 \%)$, thoracotomy was not undertaken because they had metastases when first seen, or because they were too old or too ill or declined treatment, or because they died during the course of investigation. 

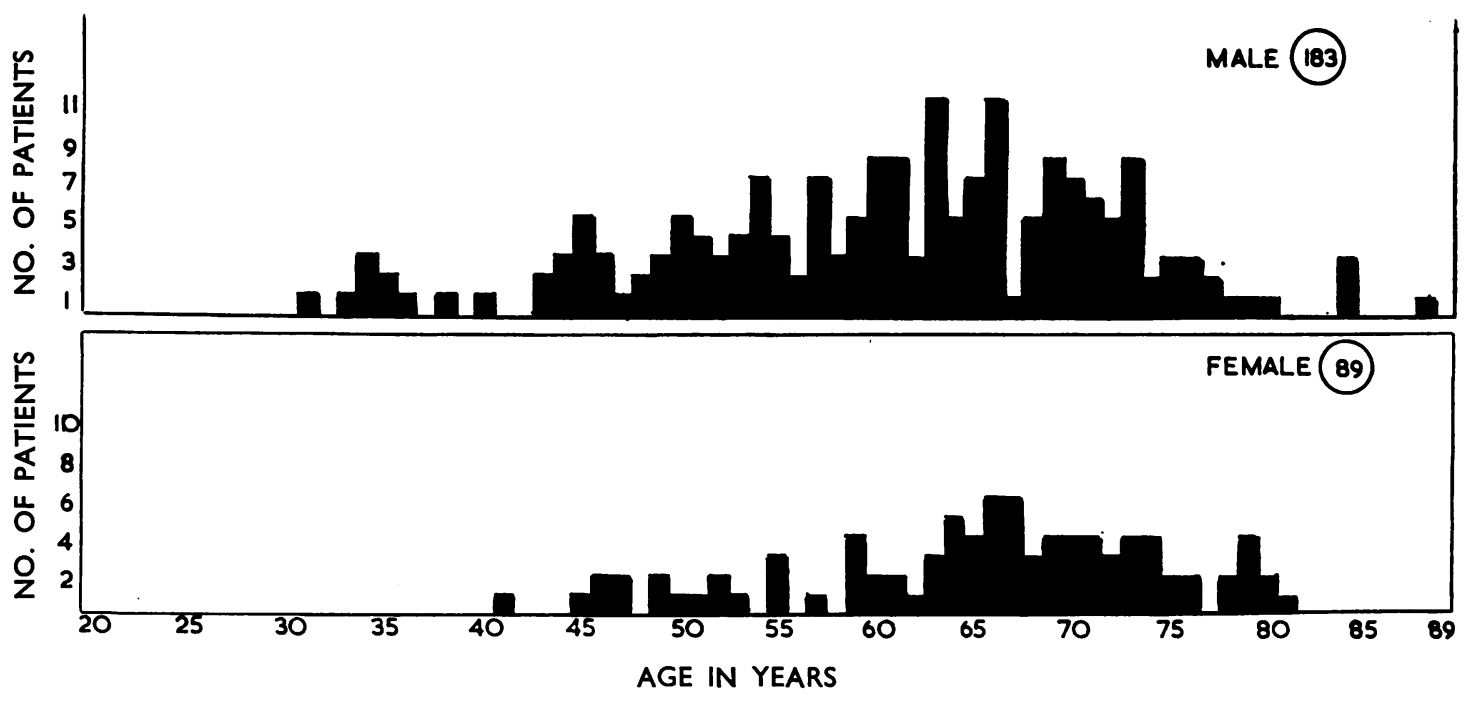

FIG. 17.

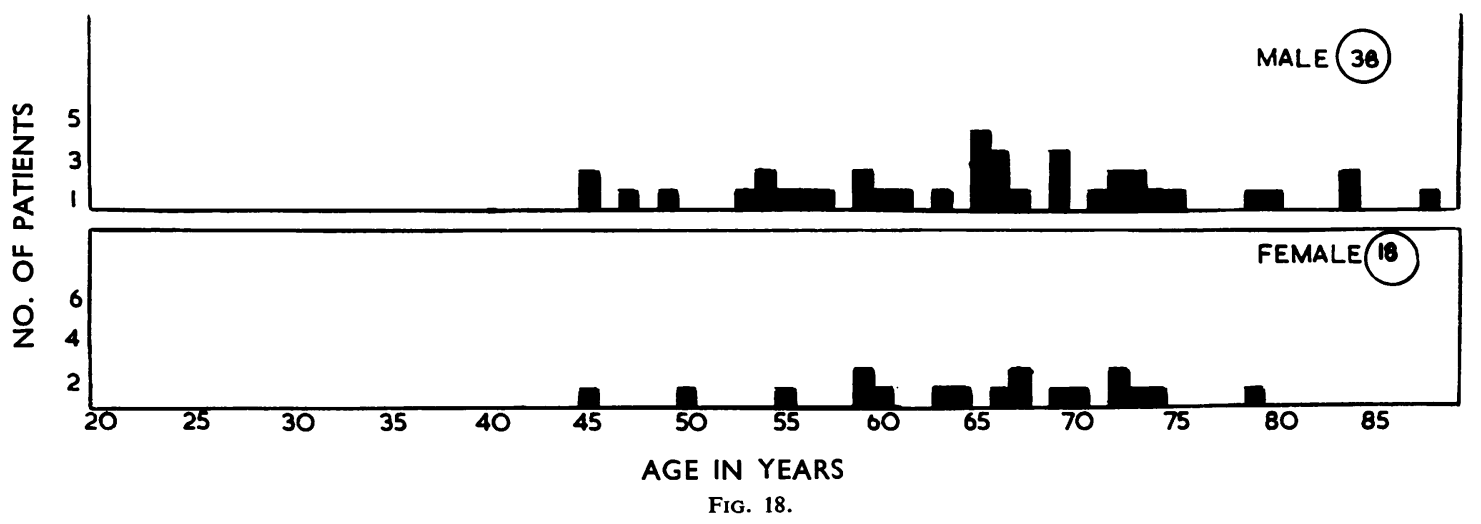

Adenocarcinoma Not Treated by Operation. -Fifty-six patients with an adenocarcinoma in the oesophagus or proximal stomach were not submitted to operation. The youngest was 45 years of age and the oldest was 89 . The age range in the two sexes is shown in Fig. 18. All these patients are dead. The tumour was visible oesophagoscopically in 26 and was beyond the range of the oesophagoscope in 30 .

All these patients had dysphagia. Since operation was not undertaken the duration of dysphagia in this group is synonymous with survival time from the onset of dysphagia (Fig. 19). Two patients died within two months of the onset of dysphagia. One survived for 26 months and the average duration of dysphagia was seven months. The average duration of dysphagia before

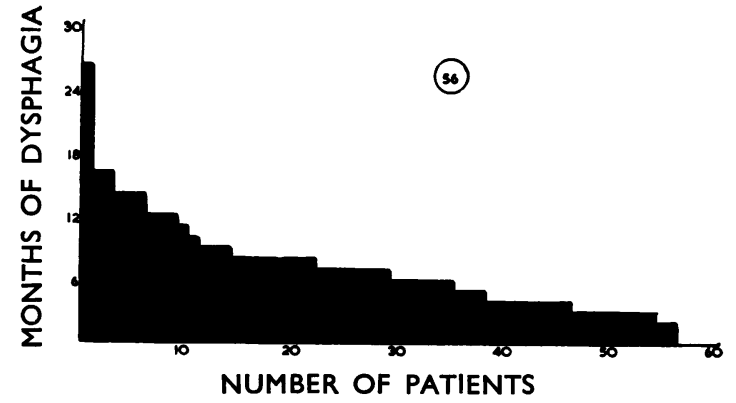

Fig. 19.

admission to hospital (Fig. 20) was between four and five months. There was no significant difference in the duration of dysphagia in those who had visible tumours and in those who did not. 


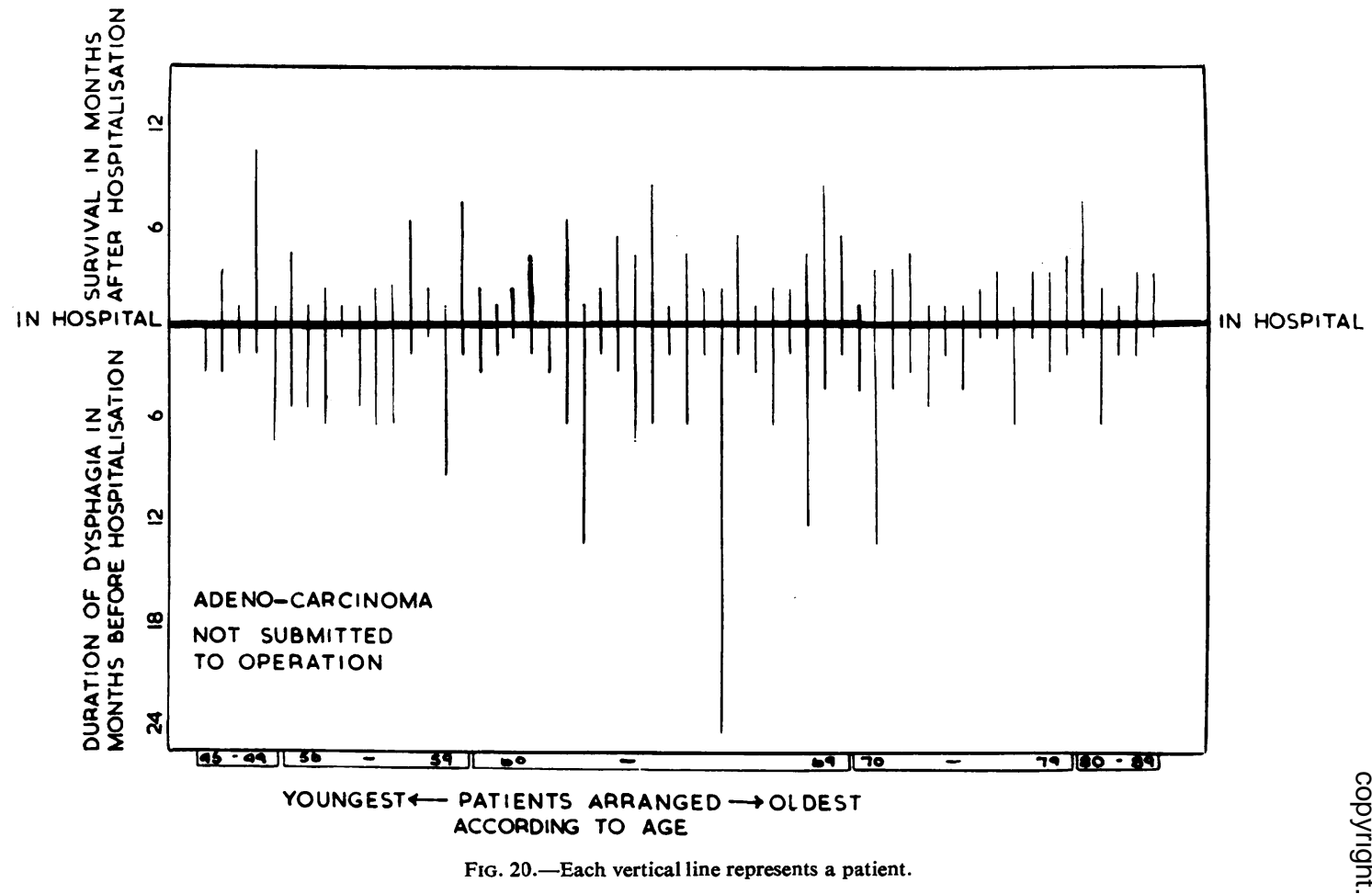

Dysphagia was the first symptom in $60 \%$ of this group, and was as often the first symptom in those who did not have visible tumours as in those who had. Forty per cent. had symptoms before the onset of dysphagia-loss of appetite and weight, vague abdominal discomfort, and in one patient haematemesis-and these symptoms preceded the onset of dysphagia by three to six months. The duration of dysphagia before admission to hospital and survival following investigation is shown in Fig. 20.

The reasons for not undertaking resection in these patients were as follows:

Fourteen patients were found clinically to have hepatic metastases and in all these the presence of hepatic metastases was confirmed at necropsy.

Fourteen patients had cervical glandular metastases, 10 on the left and four on the right; histological confirmation of spread of the tumour to cervical glands was obtained in all these cases.

Seven patients had evidence of transcoelomic spread, five presenting with ascites and two with a mass in the pelvis felt on rectal examination.

Two patients had histologically proven cutaneous metastases.

One patient had metastases at the umbilicus.
One patient presented with hoarseness of voice in addition to dysphagia and in him the left recurrent laryngeal nerve was interrupted.

Three patients had radiological evidence of diffuse pulmonary metastases and in two of these this diagnosis was later confirmed at necropsy.

Three patients died during investigation from massive bleeding from a fundal carcinoma; at necropsy all had evidence of widespread dissemination of the tumour.

One patient died from pulmonary embolism during the course of investigation; in her the tumour was seen to have invaded the posterior abdominal wall at necropsy.

Eight patients were not submitted to operation because of their advanced years, their poor general condition, or because of so limited a respiratory reserve that they were thought unable to tolerate thoraco-laparotomy.

Two patients declined treatment.

Of the 26 endoscopically visible tumours, 22 were visible at the distal end of the oesophagus, between 35 and $41 \mathrm{~cm}$. from the upper alveolus. In one patient, in addition to the distal tumour, adenocarcinoma was obtained from a biopsy taken from a nodule at the level of the aortic arch; 


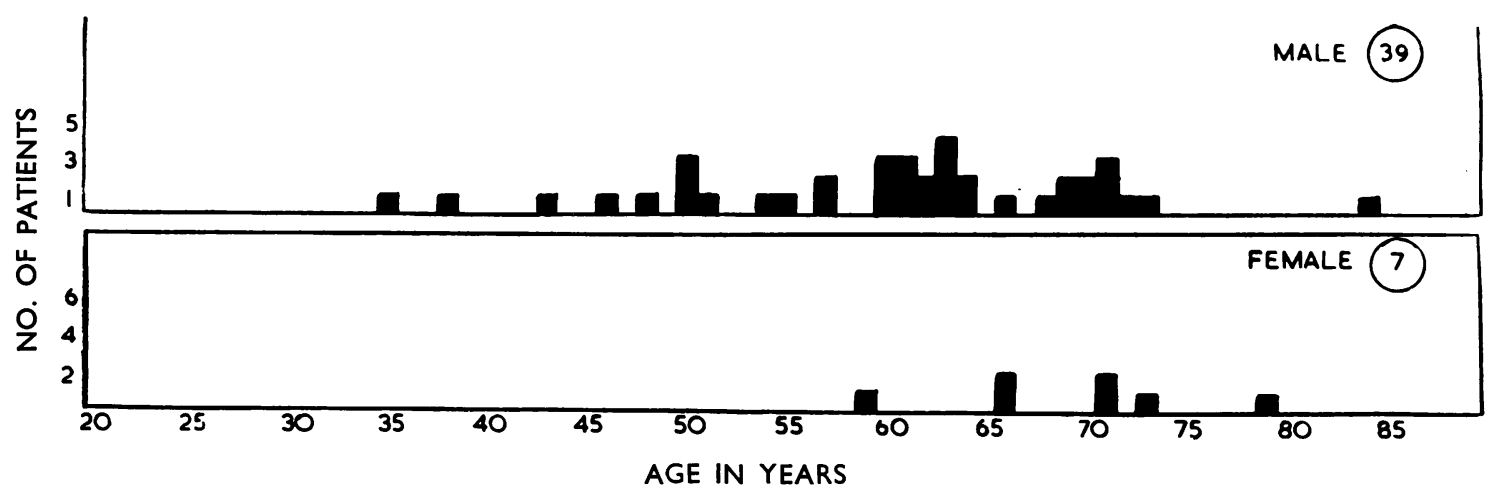

FIG. 21.-Age and sex distribution of patients with tumours visible and not visible endoscopically.

this was regarded as evidence of submucous lymphatic extension of the tumour since it was separated by normal squamous epithelium from the more distal tumour. In four patients the tumour was seen endoscopically 24 to $27 \mathrm{~cm}$. from the upper alveolus. In these patients there was radiographic evidence of a hiatus hernia, and at necropsy the tumour was growing in a viscus which had the appearances of stomach while lying in the mediastinum.

Only one patient was irradiated in this group; resection was not undertaken because his respiratory reserve was so small, and he died a year after the onset of dysphagia, five months after completing a course of radiotherapy. The tumour was, intubated with a Souttar's or Simmonds' tube in 12 of those with endoscopically visible tumours to enable liquids to be swallowed. Tumours in the gastric fundus are rarely suitable for intubation, but these patients seldom have total dysphagia.

Information regarding the mode of death in those who died at home is not accurate, but many appear to have died from the general effects of widely disseminated malignant disease rather than from some specific manifestation of the tumour, such as aspiration pneumonia because of total obstruction of the oesophagus or invasion of the respiratory tract. No patient was discarded from operation because of evidence of invasion of the trachea or main bronchi, while this was the commonest single reason for not undertaking an operation in patients with squamous carcinoma of the oesophagus.

Fifty-six patients with adenocarcinoma of the oesophagus were not submitted to operation. The average survival from the onset of dysphagia was again seven months; hepatic and glandular metastases were the commonest reasons for not undertaking resection.
AdENOCARCINOMA Found IRRESECTABLE AT THORACO-LAPAROTOMY.-Forty-six patients (seven women and 39 men) who were submitted to thoraco-laparotomy were found to have an irresectable tumour. In 24 the tumour had been visible endoscopically; in 22 the oesophagus was normal and the operation was undertaken because of the symptom of dysphagia in conjunction with barium evidence of a tumour in the gastric fundus.

The age and sex distribution of the patients in whom the tumour was endoscopically visible and of those in whom the tumour was not visible are shown in Fig. 21.

Visible Endoscopically.-In those patients with endoscopically visible tumours resection was not undertaken because of extensive hepatic metastases in six, because of diffuse peritoneal spread in six, and because of both diffuse peritoneal spread and hepatic metastases in four. The tumour was fixed to the posterior abdominal wall in two and in six invasion of the aorta and the lung precluded resection. In one, a man of 50 , thoraco-laparotomy was undertaken in the presence of known cervical glandular metastases in an attempt to restore swallowing.

To restore swallowing, the tumour was bypassed in six patients, by the use of jejunum in five and of stomach in one. Five of the six in whom a bypass was made died from operation (Fig. 22a), four of these from leakage from the anastomosis and one from respiratory infection. Two others died as a direct consequence of operation, both from respiratory infection. The operative mortality in this group of 24 patients is $29 \%$. Swallowing was restored in the patient who survived a bypass procedure. This was the man with cervical glandular metastases and he died two months after operation from progress of disease. In the youngest patient in this group, a 


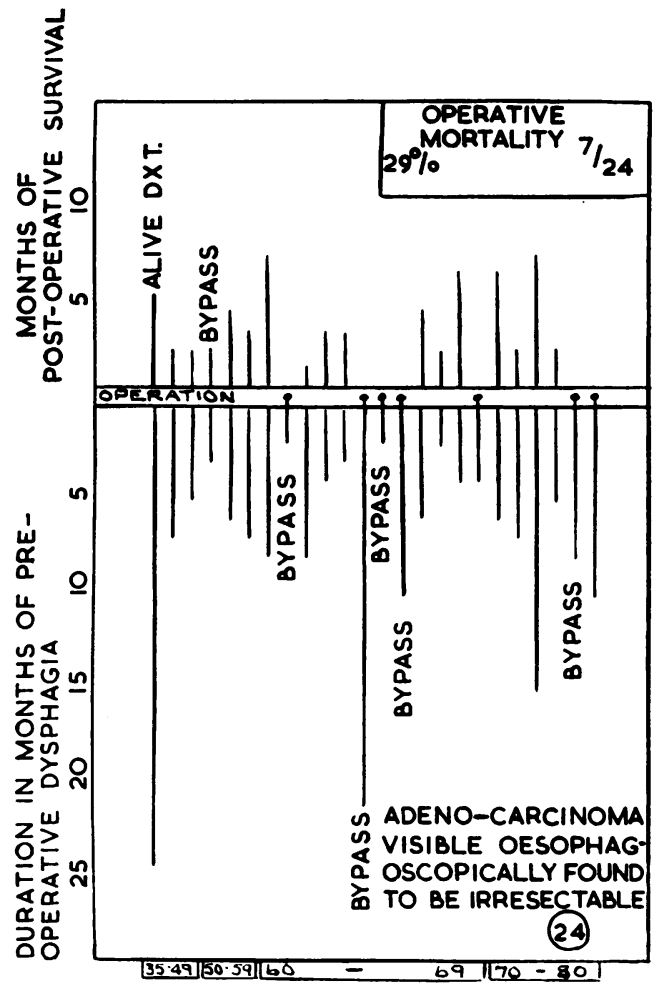

(a)

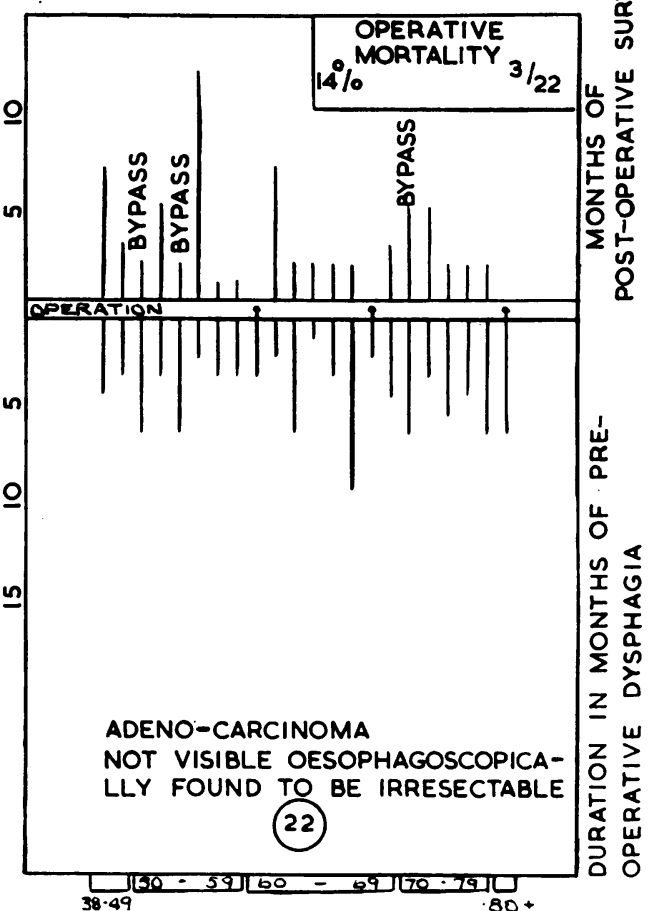

(b)

Fig. 22.-Each vertical line represents a patient, and these are arranged youngest on the left, oldest on the right. The dots in the transverse gap labelled operation represent patients who have died as a consequence of operation.

man of 35 , swallowing was restored by irradiation after thoraco-laparotomy and this patient is still alive seven months after thoraco-laparotomy. Of the patients who survived operation, later to die from progress of disease, none survived longer than seven months. The duration of pre-operative dysphagia and post-operative survival in this group of patients is shown in Fig. 22a. In the 23 patients who have died dysphagia had been present in two for only two months; both of these were operative deaths. One patient survived 22 months from the onset of dysphagia (Fig. 23a). The average survival from the onset of dysphagia was nine months. The average duration of dysphagia before operation was seven months. The patient still alive has survived 31 months from the onset of dysphagia.

Not Visible Endoscopically.-In those patients in whom the tumour was not visible endoscopically resection was not undertaken because of extensive hepatic metastases in six, because of diffuse peri- toneal spread in nine, and because of both diffuse peritoneal spread and hepatic metastases in five. In two patients fixation of the tumour to the posterior abdominal wall precluded resection. There was no intrathoracic contraindication to resection. In three swallowing was restored by bypassing the tumour with jejunum (Fig. 22b). Two of these survived for two months and one for five months after operation, dying then from progress of disease but still able to swallow. Three died as a consequence of operation, two from respiratory infection and one from an undetermined cause, an operative mortality in this group of 22 patients of $14 \%$. All these 22 patients are dead. One survived only two months from the onset of dysphagia, dying as a consequence of operation. The longest survivor lived for 14 months from the onset of dysphagia (Fig. 23b). The average survival from the onset of dysphagia was seven and a half months. The average duration of dysphagia before operation was four months. 


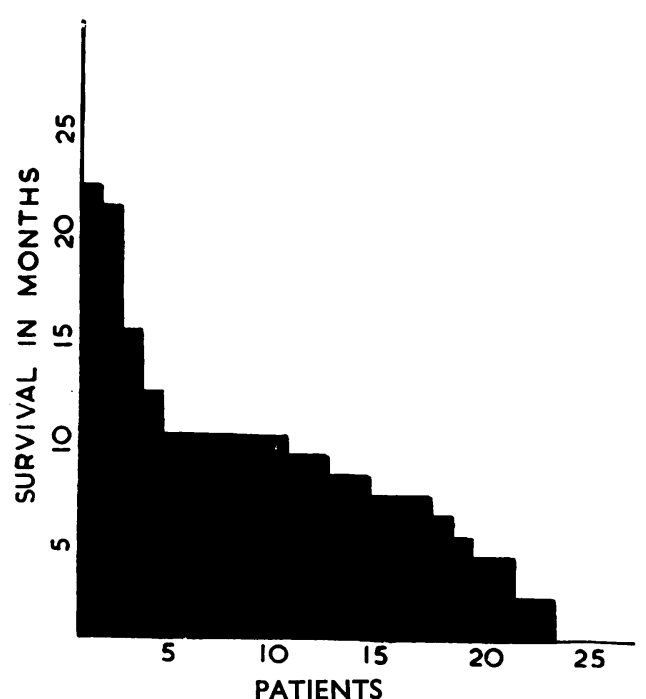

(a)

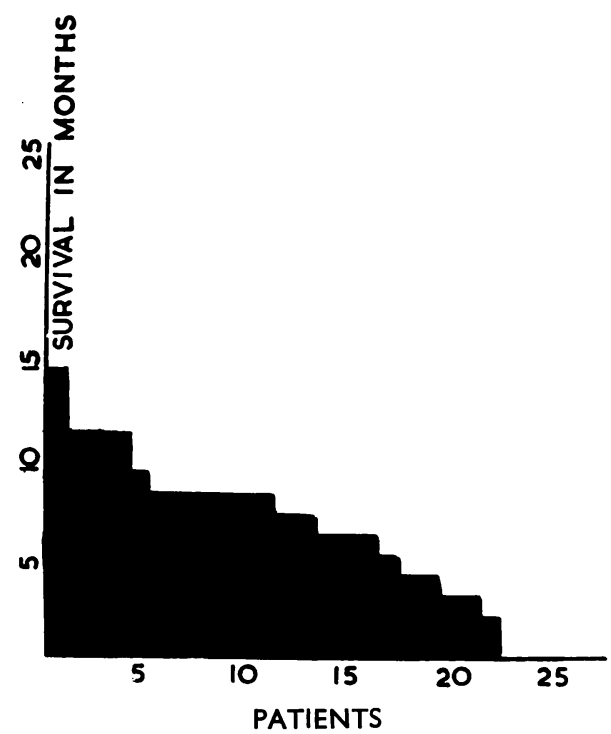

(b)

FIG. 23.

Three of the patients with oesophagoscopically visible tumours found irresectable at thoracolaparotomy had large hiatus herniae and in these the tumour was visible endoscopically at the lower border of the aortic arch. In one patient in whom the tumour was visible at $22 \mathrm{~cm}$. from the upper alveolus there was no evidence of hiatus hernia at thoracotomy, but there was a very large tumour extending from the gastric fundus to above the aortic arch and invading the aorta.

To sum up, 46 patients with an adenocarcinoma in the oesophagus or proximal stomach were found at thoraco-laparotomy to have tumours unsuitable for resection. The operative mortality was $22 \%$. The average survival from the onset of dysphagia was a little longer than in those not submitted to operation.

\section{Adenocarcinoma Resected}

Not Visible Oesophagoscopically.-In 40 of the 170 patients in whom an adenocarcinoma was resected the tumour was not visible oesophagoscopically. Sixteen of these were women and 24 were men. The age incidence in the two sexes is shown in Fig. 24. The average duration of dysphagia in these 40 patients was five months, not significantly different from the duration in those presenting initially with a contraindication

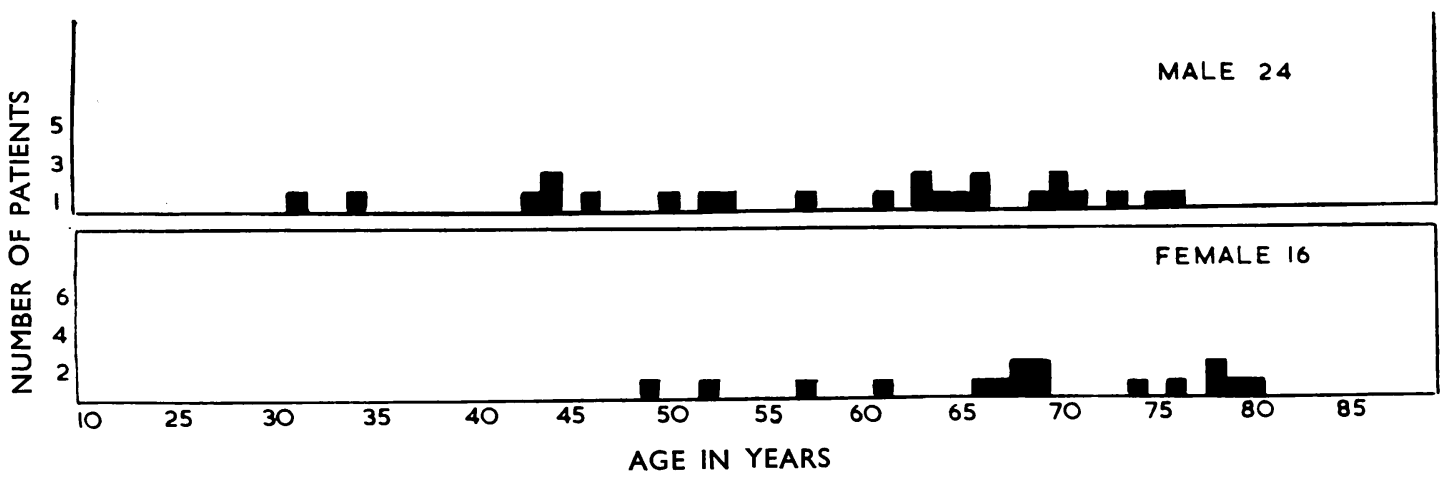

FIG. 24. 


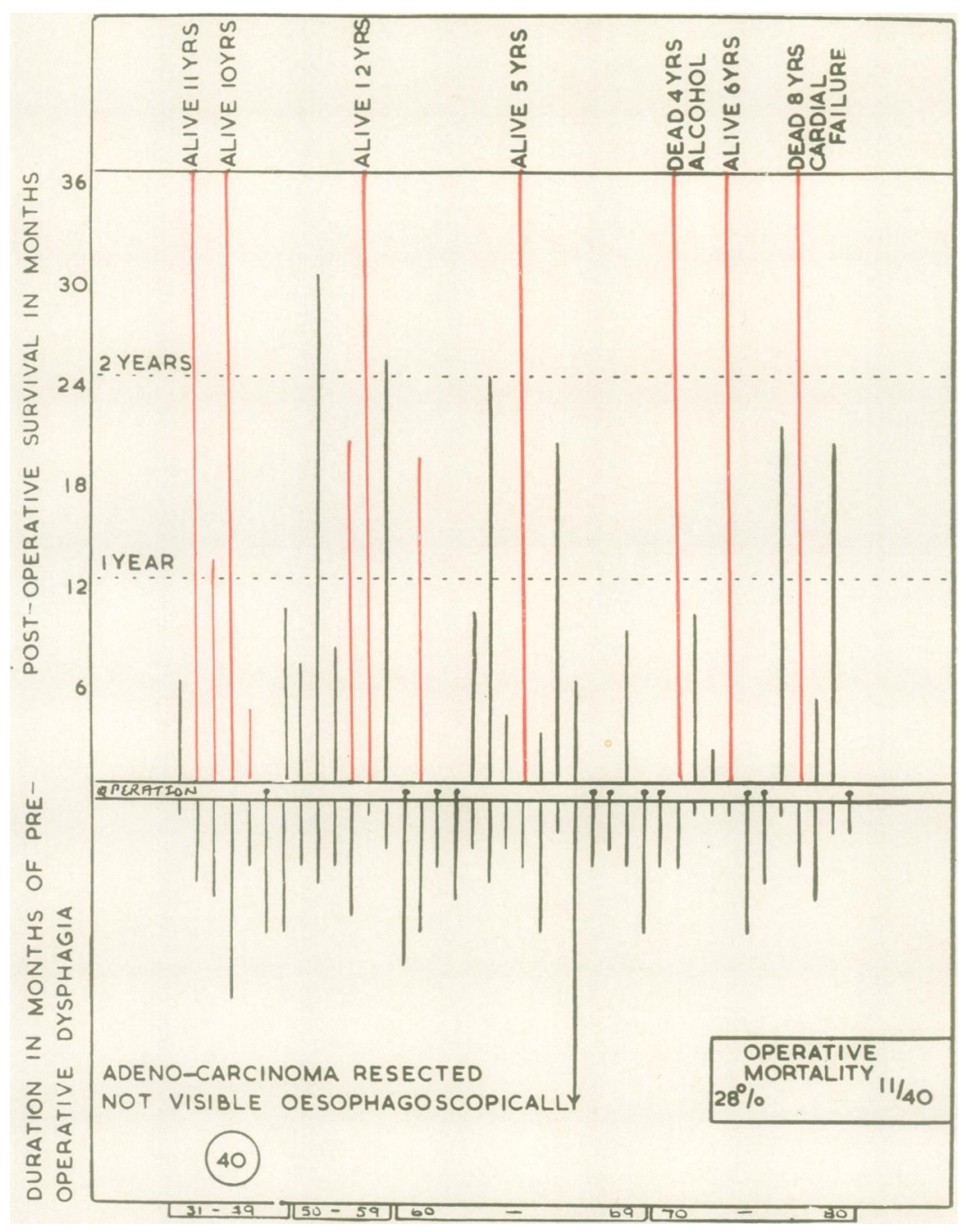

FIG. 25.-Each vertical line represents a patient, and these are arranged the youngest on the left and the oldest on the right. The dots in the transverse gap labelled operation represent patients dying from operation. Vertical red lines represent patients at present alive or patients who have died from causes other than metastases more than three years after operation. The vertical black lines above the operative gap represent patients who have died from metastases.

to resection or those found unsuitable for resection at thoraco-laparotomy. An oesophago-gastrostomy was made in 19 of these and an oesophagojejunostomy in 21.

Eleven patients died as a consequence of operation, an operative mortality of $28 \%$ (Fig. 25). Four of these patients died because of disruption of the anastomosis, in all four instances an oesophago-jejunostomy. Three patients died from respiratory infection. Two died within 24 hours of operation and their behaviour during this period suggests that they died from surgical "shock." They remained hypotensive throughout the short period of post-operative survival and failed to respond to resuscitative measures. One patient died 10 days after operation with a right chylothorax which probably contributed significantly to her death. One died from intestinal obstruction; the obstruction was to the ante-colic loop of jejunum used to re-establish continuity. 
Resection was undertaken in six patients who were found at operation to have hepatic metastases, in three with peritoneal metastases and in a further three with metastases in the omentum. In these 12 histological evidence of spread was obtained. It is anomalous to have abandoned resection in many described in the previous section because of hepatic or peritoneal metastases, and yet to undertake resection in the presence of these manifestations of spread in the present series of 40 patients. The anomaly is explained by the severity of the dysphagia in a few patients in whom resection was undertaken-some of them were unable to swallow even liquids without difficulty - and by the extent of neoplastic spread in others, resection having been undertaken in the presence of a single small hepatic metastasis and abandoned because of multiple large hepatic metastases.

In 20 patients in whom resection was undertaken glandular metastases were found either in the abdomen or in the mediastinum. Since two had both hepatic and glandular metastases resection was undertaken in only 10 patients who were not shown at the time of operation to have spread of the tumour beyond the stomach, i.e., $75 \%$ of patients had evidence of dissemination.

Twenty-nine patients survived operation (Fig. 25). Eighteen of these died subsequently from metastases, 12 in the first year after operation, four in the second year, and two in the third year. Fourteen of these 18 patients were shown at operation to have metastases and four were believed not to have metastases at the time of operation. These four died respectively with cerebral metastases, with a recurrence in the mediastinum producing dysphagia, and two with hepatic metastases. In four of the 18 patients dying from metastases dysphagia recurred before death six, 21, 24, and 31 months after operation.

Nine patients are alive at the present time (Fig. 25). Three are alive more than 10 years after resection. Two are alive, one six and one five years after resection, and four are alive less than two years after resection. One of the patients who is alive more than 10 years after resection had glandular metastases at operation. Three of the four alive less than two years after resection had glandular metastases at the time of operation, as did the patient alive five years after resection. Four, at present alive, did not have evidence of spread at the time of operation.

Two patients have died from causes other than operation or metastases. A woman in whom resection was undertaken at the age of 78 died eight years later in cardiac failure. She did not have metastases at the time of operation. A man in whom resection was undertaken at the age of 70 died four years later from a combination of alcoholism and bronchopneumonia. He had not been shown to have metastases at the time of operation.

All these 40 patients had had dysphagia before operation. Other symptoms such as loss of weight and appetite and abdominal pain had preceded dysphagia in 30 of them, in one by a year and in the others from three to six months. Hiccup had been a symptom in seven before their admission to hospital. One woman had pernicious anaemia.

The average survival from the onset of dysphagia in the group of 29 patients dying either as a consequence of operation or later from metastases was 12 months, the longest survivor dying 36 months from the onset of dysphagia (Fig. 26a). The average survival from the onset of dysphagia of the 18 patients who died after operation from metastases was 16 months (Fig. 26b). Whether or not operative deaths are included when calculating survival from the onset of dysphagia in those in whom a resection was undertaken and who are not at present alive or who did not die from some other cause, the survival in this group from the onset of dysphagia is longer than the seven months in those who were not submitted to operation or the nine months in those in whom the tumour was found irresectable, and is significantly longer in those dying from metastases. Of the 40 resections, 25 were undertaken more than five years ago, and of these 25 patients seven died from operation. The five-year survival rate amongst the patients who may so far have had the opportunity of surviving five years is therefore $24 \%$ of those submitted to operation and $33 \%$ of those surviving operation.

In summary, 40 adenocarcinomas not visible through the oesophagoscope were resected.

Eleven patients $(28 \%)$ died as a direct consequence of operation.

Eighteen patients $(45 \%)$ died from metastases three to 36 months after operation, an average post-operative survival of 16 months. In four dysphagia recurred.

Six patients $(15 \%)$ were alive five years after operation, and one of these died eight years after operation from an unrelated cause.

One patient died four years after operation from an unrelated cause.

Four patients are still alive, but less than two years after resection. 


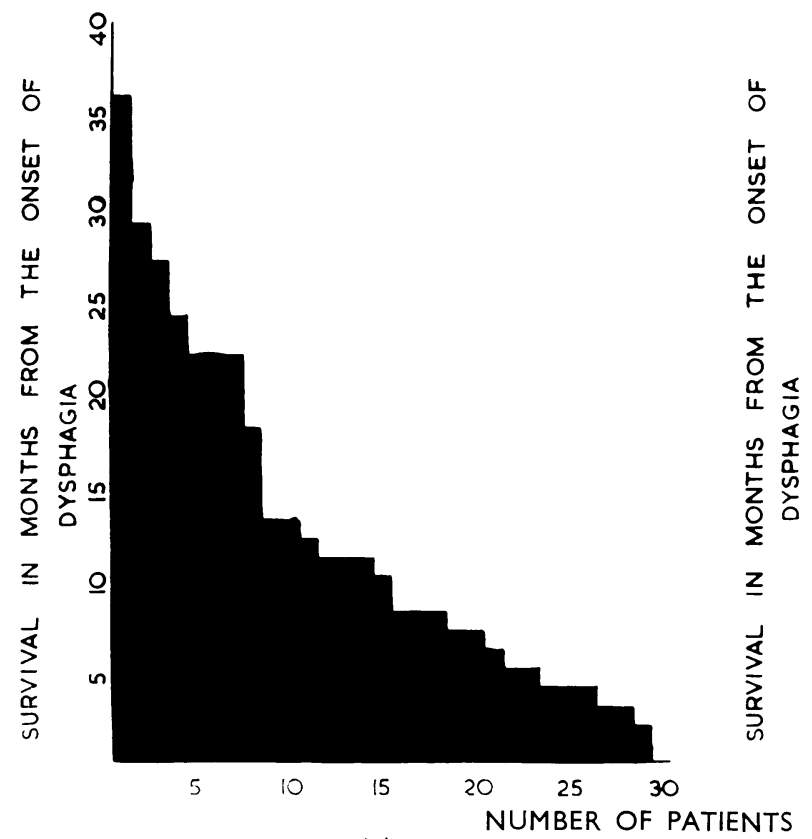

(a)

FIG. 26.

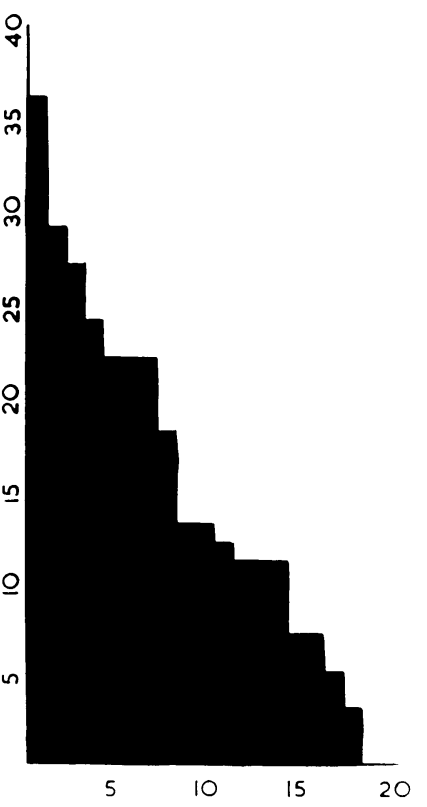

(b)
Visible Oesophagoscopically. - One hundred and thirty of the 170 adenocarcinomas resected were visible oesophagoscopically, 48 in women and 82 in men. The average duration of preoperative dysphagia in these 130 patients was a little less than five months: nearly six months in the women and just over four months amongst the men. Here again there is no significant difference in the duration of pre-operative dysphagia from its duration in those in whom a contraindication to thoracotomy was found when first they presented or in those found to be unsuitable for resection at thoraco-laparotomy. The age incidence amongst the two sexes is shown in Fig. 27.

The Operation.-An oesophago-gastrostomy was made in 108 patients and an oesophagojejunostomy in 22. In all but three the approach was through a left thoraco-laparotomy. In all but four of those approached from the left the anastomosis was made at a level which varied from the lower pulmonary vein to the lower border of the aortic arch. In four patients in whom a left thoraco-laparotomy was made the

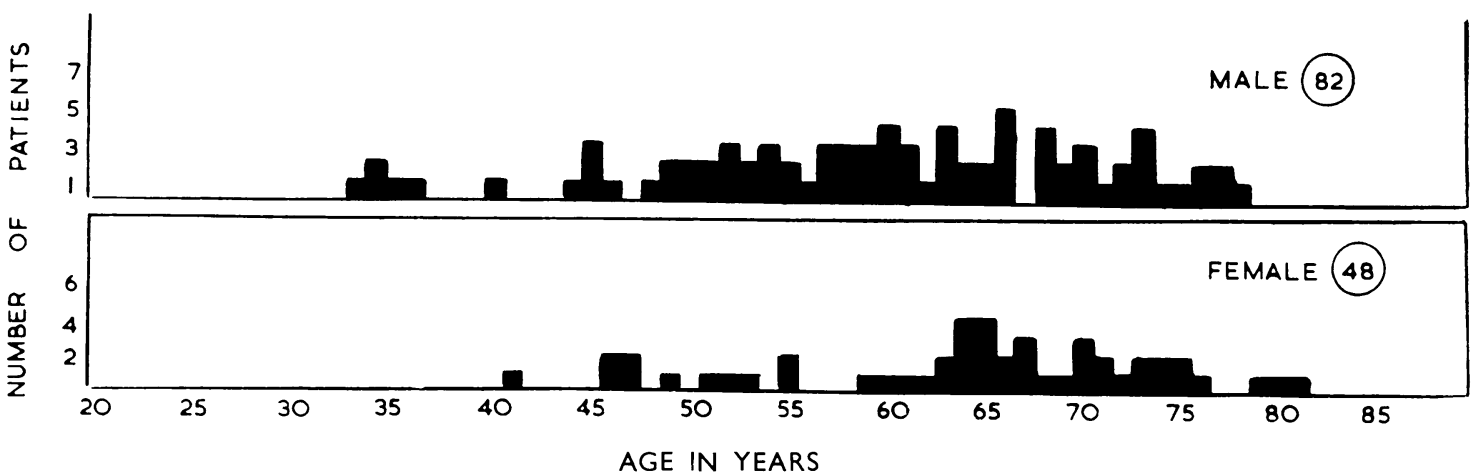

FIG. 27. 
stomach was brought up lateral to the aortic arch and a supra-aortic oesophago-gastrostomy was made. A right thoraco-laparotomy was made in three patients and in these the oesophago-gastrostomy lay above the level of the azygos vein. Seven of the 130 resections may therefore be said to have been for " high" tumours, in comparison with 10 tumours lying closer than $30 \mathrm{~cm}$. to the upper alveolus amongst 50 patients with endoscopically visible adenocarcinomata either not submitted to resection because of a contraindication or found unsuitable for resection at thoracolaparotomy. In both squamous-cell carcinoma and adenocarcinoma there is a higher rate of irresectability amongst the high tumours.
Operative Mortality.-Thirty-two patients died as a consequence of operation (10 women and 22 men), an operative mortality of $25 \%$ (Figs. 28 and 29). The operative mortality amongst men (27\%) was higher than amongst women $(21 \%)$. The overall operative mortality was lower than in resection for squamous carcinoma, and the higher mortality amongst women with squamous carcinoma was here reversed. Amongst women most of the operative deaths were in the age group 60-70. Amongst men most of the operative deaths were in those over 65 and, as with squamous carcinoma, $50 \%$ of men over 70 submitted to resection died directly as a consequence of operation. None of the

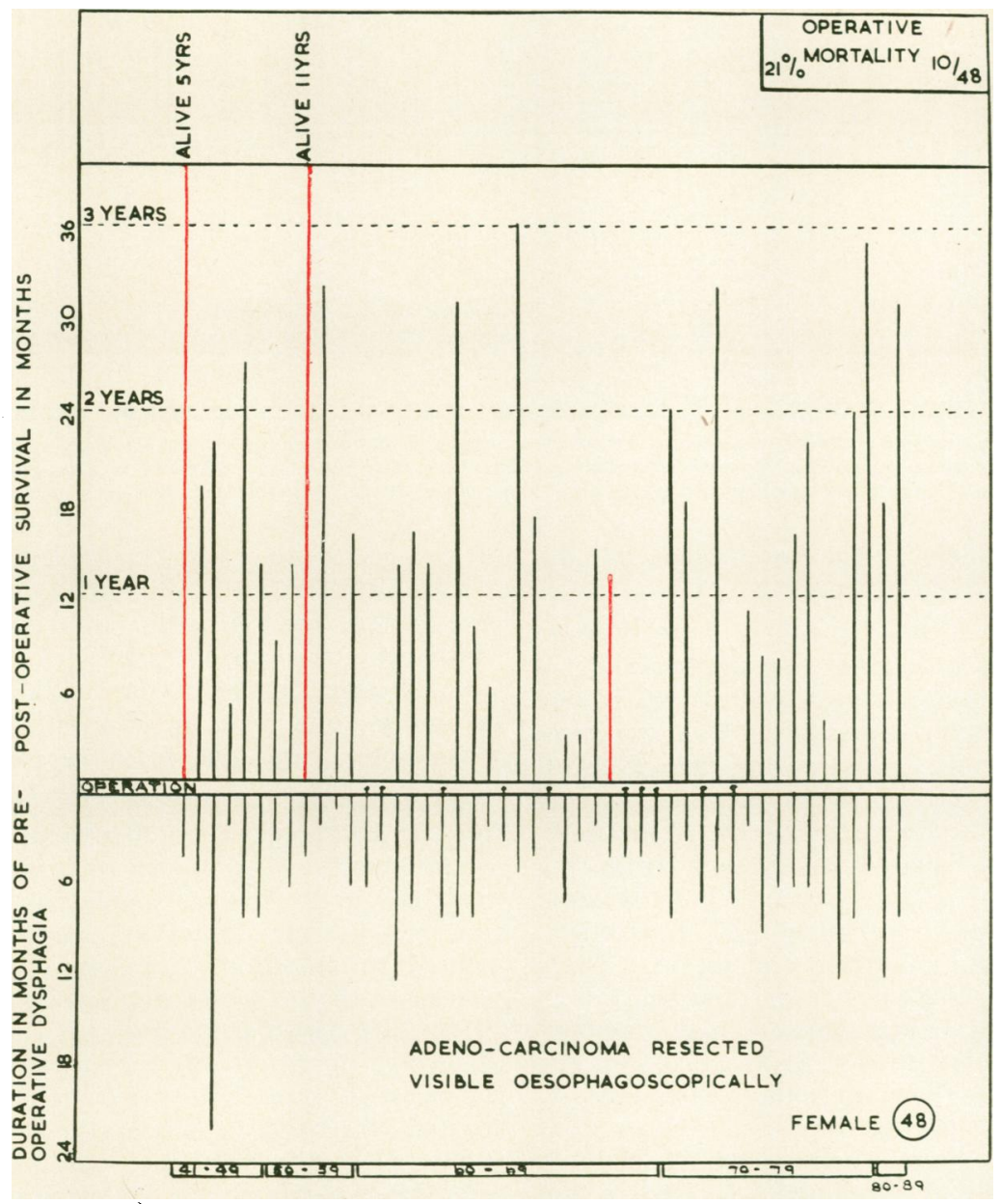

FIG. 28.-Each vertical line represents a patient, and these are arranged the youngest on the left and the oldest on the right. The dots in the transverse gap labelled operation represent patients dying from operation. Vertical red lines represent patients at present alive. The vertical black lines above the operative gap represent patients who have died from metastases. 


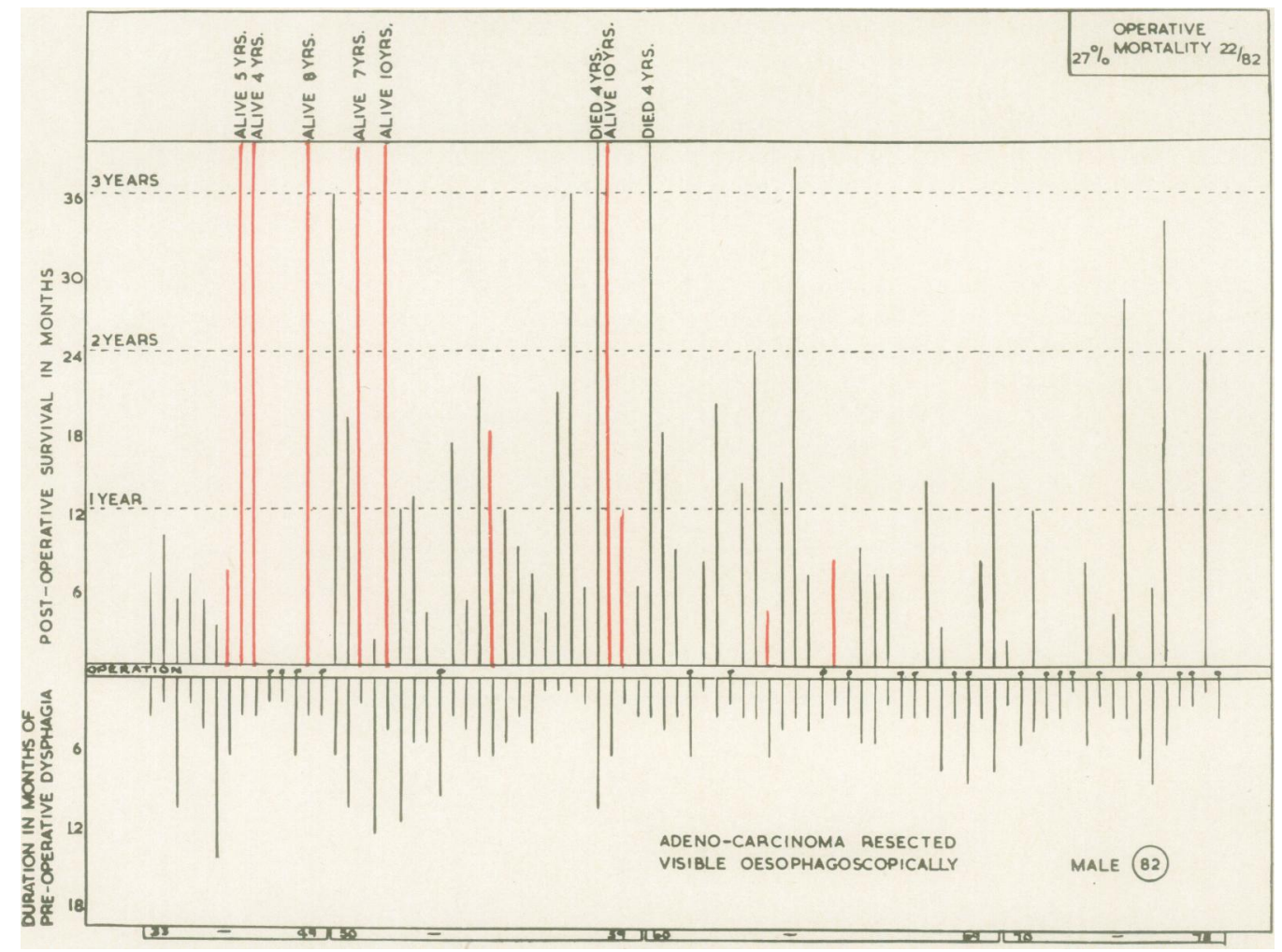

FIG. 29.-Each vertical line represents a patient, and these are arranged the youngest on the left and the oldest on the right. The dots in the transverse gap labelled operation represent patients dying from operation. Vertical red lines represent patients at present alive. The vertical black lines above the operative gap represent patients who have died from metastases.

patients with "high" adenocarcinomas of the oesophagus died from operation.

Nine operative deaths, one in a woman, were a consequence of leakage at the anastomosis; in eight from an oesophago-gastrostomy and in one from an oesophago-jejunostomy. Four patients, all men, died from haemorrhage, the result of erosion of the aorta. This complication of oesophagectomy is discussed in detail elsewhere, but it is closely related to leakage from the anastomosis. Four patients, three of them women, died from pulmonary embolism, and four others, all men, died from pulmonary infection. Three patients died as a consequence of a cardiac lesion, two from electrocardiographically and histologically established myocardial infarction, and one in cardiac failure from an undetermined cause. Five patients died during the first 24 hours after operation, three from a cause which includes operative shock (they remained hypotensive and failed to respond to resuscitative measures) and two from reactionary haemorrhage, in one of these from an inadequately ligated left gastric artery and in one from an undetermined source. Three patients died from infection: one from a pyocyaneus peritonitis during a period when this organism was uncontrolled in the unit, later necessitating closure of the operating theatre for a month; one from an empyema with an intact anastomosis, and one from necrotizing enteritis.

Metastases at Operation.-In 83 patients (64\%) histological confirmation of glandular metastases in the abdomen or in the mediastinum was obtained. Twenty patients in whom hepatic metastases were found at operation were submitted to resection to restore swallowing, and seven of these 20 did not have glandular metastases. Peritoneal spread was found in three, extensive invasion of the right lung in two, invasion of the transverse colon necessitating partial colectomy in one, and in two macroscopic tumour was left in the mediastinum. All the lastmentioned eight patients had, in addition, glandular metastases. Seventy per cent. of patients with endoscopically visible adenocarcinomata undergoing resection had dissemination at operation, 
resection being undertaken in only 40 out of the total of 130 in the absence of histologically proven dissemination of tumour, and eight of these 40 died as a consequence of operation. Ninety-eight survived operation, 32 of these without evidence of spread of the tumour at operation. Of these 32,23 died subsequently from metastases and nine are alive at the present time.

SYMPTOMS: OPERATION UNDER UNUSUAL CiRCUMSTANCES.-All of the 130 patients with endoscopically visible adenocarcinomas had dysphagia before operation. Other symptoms, such as loss of weight and appetite, had preceded dysphagia in 45 , a number significantly smaller than amongst the adenocarcinomata not visible endoscopically. In two patients symptoms other than dysphagia had preceded dysphagia by a year ; in the remainder other symptoms preceded dysphagia by three to six months. Hiccup had been a symptom in eight before admission. In four men there was a long history of intermittent dysphagia -15 to 25 years - with a recent change in the character of the dysphagia. In one of these the radiographic appearances were those of longstanding cardiospasm, and in the other three there were the radiographic and operative features of an hiatus hernia; in these three patients the carcinoma may have developed in relation to a benign peptic stricture at the oesophago-gastric junction. In six others there was a large hiatus hernia visible radiologically and demonstrated with barium.

In a man of 55 resection was undertaken to restore swallowing despite the presence of histologically established cervical glandular metastases. This man died 22 months after resection, and dysphagia recurred three months before death; he had swallowed normally and lived his normal life for 18 months after resection. One woman was shown pre-operatively to have an unusually large left pericardial coelomic cyst.

RADIOTHERAPY.-Two men were referred for resection after irradiation. One had been explored through the abdomen elsewhere in 1955, and had been regarded as having an irresectable tumour which was irradiated with some relief of dysphagia. Dysphagia became complete in 1957, and the tumour was resected in the presence of mediastinal glandular metastases. In January, 1960 , he developed intestinal obstruction, the consequence of herniation of small gut through the gastric hiatus in the diaphragm; the obstruction was relieved and the hiatus repaired, and he is well and swallows normally. No evidence of spread was found at this last operation. Experi- ence with this man and others, explored through the abdomen and regarded as having irresectable tumours, most of them not irradiated, and later referred for the relief of total dysphagia, has confirmed the contention that proximal gastric tumours cannot be properly assessed through a purely abdominal approach. The other patient whose tumour was irradiated before resection was young (36 years) and was regarded elsewhere as having an irresectable tumour without exploration.

In him irradiation improved swallowing for a year. When dysphagia became intolerable the tumour was resected but he died from metastases five months later. One patient was irradiated for recurrence of dysphagia 30 months after resection and survived irradiation by only three months without relief of dysphagia.

Non-fatal Complications.-Major post-operative complications with survival were few. Two subphrenic abscesses and one empyema were drained. One patient developed clinical and radiographic evidence of leakage from the anastomosis into the right pleural space; the pleural space was intubated, and methylene blue taken by mouth appeared rapidly in the waterseal. This patient was managed conservatively; hydration was maintained by venoclysis for some days and then a jejunostomy was made; he survived and is still alive 10 years after resection, being one of three survivors from clinically recognized leakage from the anastomosis. One man, aged 45, an unusually muscular individual and an active mountaineer, six months after oesophago-gastrectomy with gastric replacement, developed intermittent severe substernal pain relieved by vomiting; these symptoms were related to exercise. He was shown to have a mobile intrathoracic gastric tube and his symptoms were attributed to recurring torsion of this tube. The stomach was fixed at a second operation, at which no metastases were found, and he is well six years after his original resection.

The Fate of those who Survived Operation. -Ninety-eight patients survived operation, 32 of these without dissemination of tumour at the time of operation. Eighty-two patients, 47 men and 35 women, died subsequently from metastases. Two men died during the fourth year after operation from non-metastatic causes; 11 men and three women are at present alive.

Of the 82 patients ( 29 men and 12 women) who died from metastases after resection, 41 died during the first post-operative year (Figs. 28 and 29). During the second year after resection 29 patients (13 men and 16 women) died from meta- 


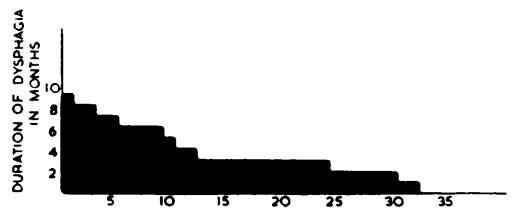

(a)

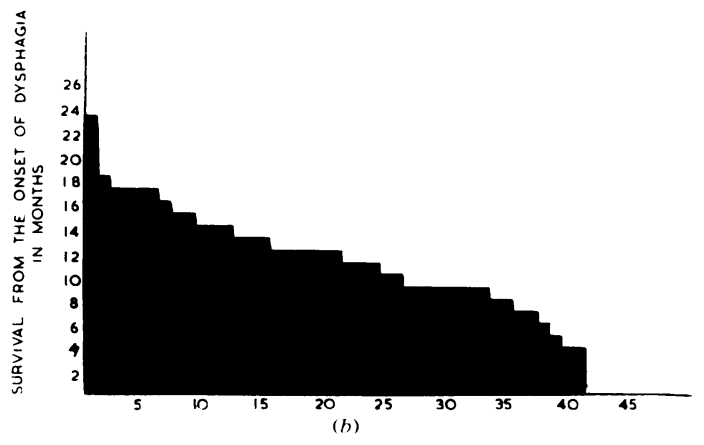

NUMBER OF PATIENTS

FIG. 30.

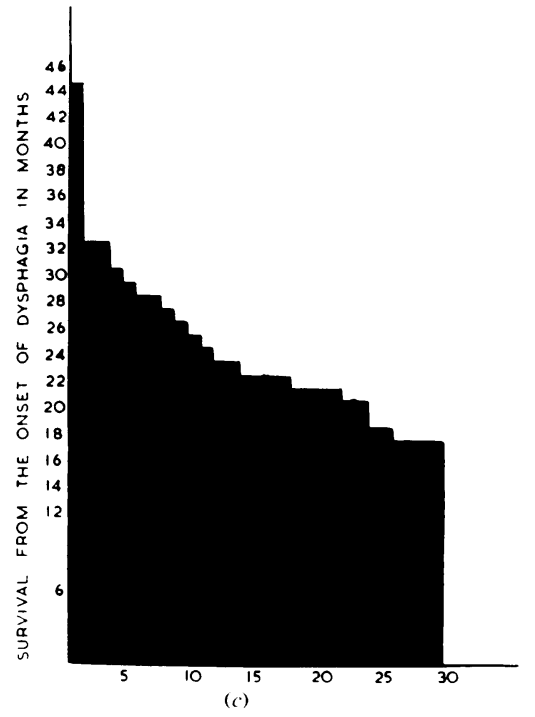

(c) stases, and during the third year after resection 11 patients (four men and seven women) died from metastases. One man died from metastases during the fourth year after resection, and two other men died during the fourth year after resection, one from cerebral thrombosis and one from coronary thrombosis. In both these there had been spread at the time of resection.

Eleven men and three women are alive at the present time. Of these 14 patients, four are alive a year or less following resection, and two others are alive less than two years after resection. Five of the six alive less than two years after resection had glandular metastases. One man is alive four years after resection and seven patients (five men and two women) are alive five years or more after resection. Two of these have very nearly completed their fifth year after resection, one is alive seven years and one eight years after resection. two are alive 10 years, and one is alive 11 years after resection. None of the patients surviving five years or longer after resection had dissemination of tumour at the time of resection. Seven patients, of 61 who underwent resection and of 48 who survived resection more than five years ago, may be regarded as five-year survivors. a fiveyear survival rate of $12 " \ldots$, calculated against the number submitted to operation and of $15^{\circ}$.. calculated against the number surviving operation. Continuity was re-established in five. who have survived five years or more, using stomach, and in two using jejunum. Continuity had been re- established using jejunum in one out of every five resections.

Recurrence OF Dysphagia. - Dysphagia recurred in 20 of the 98 patients who survived operation, and all 20 had spread at the time of resection. Eight died with recurrence of dysphagia during the first year after resection, seven died with recurrence of dysphagia during the second year after resection, and one is alive during the second year after resection, but has a recurrence of tumour, and four died during the third year after resection with recurrence of dysphagia. In nearly all these patients dysphagia recurred less than two months from the time of death. and recurrence at the anastomosis was only one of several evidences of recurrence or spread in these patients. One patient alive five years after resection has a peptic stricture at the oesophagogastrostomy requiring dilatation at about yearly intervals.

Incidence of Effective Palliation.--The palliative value of resection in patients dying from metastases must be assessed against the average survival of those patients with an adenocarcinoma producing dysphagia who were not submitted to operation. In this group of 56 patients the average duration of dysphagia before investigation was shown earlier to be four to five months, and average survival from the onset of dysphagia was seven months. Thirty-two patients died from operation: in these the average duration of 
dysphagia before operation was four months (Fig. $30 \mathrm{a}$ ), and since they died from operation, average survival from the onset of dysphagia is also four months, and no increase of survival time accrued to them. Forty-one died from metastases during the first post-operative year, and of these 41, eight died with recurrence of dysphagia. Average survival from the onset of dysphagia in this group of 41 was $11 \frac{1}{2}$ months, the longest survival being 23 months from the onset of dysphagia and the shortest four months (Fig. 30b). As with squamous carcinoma, there is no increase in survival time and resection in 73 patients $(32+41)$ did not prolong life and can be regarded only as an incident in the natural course of the disease, i.e., in $56 \%$ of resections undertaken for adenocarcinoma visible through the oesophagoscope the natural history of the disease was not substantially altered. Twenty-nine patients died from metastases during the second year after resection, seven dying with recurrence of dysphagia. Average survival from the onset of dysphagia in this group was 22 months, the longest survival being 44 months and the shortest 17 months (Fig. 30c). Significant increase in the duration of survival can be claimed for these patients and for all those dying subsequently from metastases.

Summary.-One hundred and thirty oesophagoscopically visible adenocarcinomas of the oesophagus were resected.

Twenty-five per cent. of the patients died as a consequence of operation; $70 \%$ had evidence of spread at operation.

Sixty-three per cent. of those submitted to operation ( $84 \%$ of those who survived operation) died from metastases, exactly half of these during the first 12 months after resection.

Five per cent. of those submitted to operation ( $7 \%$ of those who survived operation) are alive five years or more after resection.

Seven per cent. of those submitted to operation are alive less than five years after operation or have died from non-metastatic causes.

\section{SUMMARY}

Seven hundred patients with dysphagia were found to have as the cause for this symptom a carcinoma in the proximal alimentary tract.

Twenty squamous carcinomas involved the hypopharynx ; 19 of these were in women, and of 11 patients submitted to resection four are alive five years later.

Three hundred and eighty-two patients with squamous carcinoma of the oesophagus were investigated and $62 \%$ were submitted to resection.
Invasion of the trachea and bronchi was the commonest reason for not undertaking resection in the remainder. Of those resected, $34 \%$ died from operation and $10 \%$ were alive five years later. In $60 \%$ of those submitted to operation, the natural course of the disease, as judged by the behaviour of those patients considered unsuitable for resection, was unaltered. The prognosis was worse in those with high oesophageal tumours.

Twenty-six patients with undifferentiated carcinomas were investigated, 12 were submitted to resection and one of these survived more than five years.

Two hundred and seventy-two patients with adenocarcinomas in the oesophagus or proximal stomach were investigated and $62 \%$ of these were submitted to resection. Hepatic and glandular metastases were the commonest evidences of spread which precluded resection in the remainder. Of 40 patients with tumours in the gastric fundus who were submitted to resection, six were alive five years after operation, 11 having died from operation. Resection was undertaken in 130 patients with an adenocarcinoma visible in the oesophagus ; $25 \%$ died from operation, and $5 \%$ were alive five years later. In $56 \%$ of visible adenocarcinomas resected the natural course of the disease was unaffected. Of all patients with adenocarcinoma submitted to resection, $8 \%$ were alive five years after resection.

Four hundred and eighteen oesophagogastrectomies were undertaken. The operative mortality was $30 \%$ and anastomotic failure accounted for nearly one-third of these deaths.

Of the 418 resections, 220 were undertaken more than five years ago, and, of these 220 patients, 60 died from operation. The five-year survival rate amongst those who may have had the opportunity of surviving five years is $17 \%$ of those submitted to operation and $23 \%$ of those who survived operation. Twenty-nine per cent. of the 418 patients submitted to resection or $37 \%$ of those who survived resection died from metastases during the first post-operative year. Effective palliation was achieved in $33 \%$ of those submitted to operation or $43 \%$ of those who survived operation. Forty per cent. of those submitted to resection derived significant or lasting benefit from operation, and $60 \%$ did not. Dysphagia due to recurrence developed in $14 \%$ of those submitted to operation, or $19 \%$ of those who survived operation.

\section{REFERENCES}

le Roux, B. T. (1962a). J. roy. Coll. Surg. Edinb. To be published. - (1962b). Brit.J. Surg. To be published. 